\title{
Basic Features of a Cell Electroporation Model: Illustrative Behavior for Two Very Different Pulses
}

\author{
Reuben S. Son • Kyle C. Smith • Thiruvallur R. Gowrishankar • \\ P. Thomas Vernier · James C. Weaver
}

Received: 13 April 2014 / Accepted: 7 June 2014/Published online: 22 July 2014

(C) Springer Science+Business Media New York 2014

\begin{abstract}
Science increasingly involves complex modeling. Here we describe a model for cell electroporation in which membrane properties are dynamically modified by poration. Spatial scales range from cell membrane thickness $(5 \mathrm{~nm})$ to a typical mammalian cell radius $(10 \mu \mathrm{m})$, and can be used with idealized and experimental pulse waveforms. The model consists of traditional passive components and additional active components representing nonequilibrium processes. Model responses include measurable quantities: transmembrane voltage, membrane electrical conductance, and solute transport rates and amounts for the representative "long" and "short" pulses. The long pulse- $1.5 \mathrm{kV} / \mathrm{cm}, 100 \mu \mathrm{s}-$ evolves two pore
\end{abstract}

Electronic supplementary material The online version of this article (doi:10.1007/s00232-014-9699-z) contains supplementary material, which is available to authorized users.

R. S. Son · K. C. Smith · T. R. Gowrishankar ·

J. C. Weaver $(\square)$

Harvard-MIT Division of Health Sciences and Technology,

Massachusetts Institute of Technology, 77 Massachusetts

Avenue, E25-213A, Cambridge, MA 02139, USA

e-mail: jcw@mit.edu

T. R. Gowrishankar

e-mail: tgowrish@mit.edu

K. C. Smith

Department of Electrical Engineering and Computer Science, Massachusetts Institute of Technology, Cambridge, MA, USA

Present Address:

K. C. Smith

Center for Engineering in Medicine, Massachusetts General

Hospital, 114 16th Street, Charlestown, MA 02129, USA

P. T. Vernier

Frank Reidy Research Center for Bioelectrics, Old Dominion

University, Norfolk, VA 23508, USA subpopulations with a valley at $\sim 5 \mathrm{~nm}$, which separates the subpopulations that have peaks at $\sim 1.5$ and $\sim 12 \mathrm{~nm}$ radius. Such pulses are widely used in biological research, biotechnology, and medicine, including cancer therapy by drug delivery and nonthermal physical tumor ablation by causing necrosis. The short pulse- $40 \mathrm{kV} / \mathrm{cm}, 10 \mathrm{~ns}-$ creates 80 -fold more pores, all small $(<3 \mathrm{~nm} ; \sim 1 \mathrm{~nm}$ peak). These nanosecond pulses ablate tumors by apoptosis. We demonstrate the model's responses by illustrative electrical and poration behavior, and transport of calcein and propidium. We then identify extensions for expanding modeling capability. Structure-function results from MD can allow extrapolations that bring response specificity to cell membranes based on their lipid composition. After a pulse, changes in pore energy landscape can be included over seconds to minutes, by mechanisms such as cell swelling and pulse-induced chemical reactions that slowly alter pore behavior.

Keywords Cell electroporation - Computational model · Electrical behavior - Poration behavior - Solute transport

\section{Introduction}

Growth of Computational Modeling in Science

Science has recently benefited from an almost explosive growth in computational modeling capability. Examples range from simulations of cosmic supernova, planetary system formation, climate change, weather forecasting, multi-scale modeling of molecular reactions, and system models for electrical interactions within biological systems. Here we focus on a subset of the latter. At one extreme, there are striking advances in modeling at the whole and 
partial body levels using voxels with continuum properties that are either fixed or responsive to local fields (Benkler et al. 2008; Nagaoka et al. 2008; Christ et al. 2010). At the other extreme, there are tremendous advances in modeling voltage-gated protein channels in lipid membranes, based on molecular dynamics (MD) (Shaw et al. 2010; Jensen et al. 2013).

Continuum Planar Membrane and Cell Electroporation Modeling

The first electroporation (EP) models treated artificial planar bilayer membranes, which were also the subject of experimental studies that revealed basic behavior such as reversible electrical breakdown (REB). It began with simple, 1D descriptions of planar bilayer membranes (Litster 1975; Taupin et al. 1975; Abidor et al. 1979; Sugar 1981; Weaver and Mintzer 1981; Barnett and Weaver 1991; Weaver and Chizmadzhev 1996). Cell EP modeling was preceded by passive cell models that established basic features of responses to small fields (Pauly and Schwan 1959; Kotnik and Miklavčič 2000; Foster 2000; Yao et al. 2005; Kotnik and Miklavčič 2006). Modeling has now progressed to provide approximate descriptions of singlebilayer outer plasma membranes (PMs) (DeBruin and Krassowska 1999a, b; Stewart et al. 2004; Krassowska and Filev 2007; Talele et al. 2010; Smith et al. 2013). Some continuum models of cell membrane EP involve explicit representations of additional, intracellular organelle membranes (Gowrishankar et al. 2006; Smith and Weaver 2008; Esser et al. 2010; Retelj et al. 2013). Other multicellular membrane models represent several irregular shaped cells in close proximity that approximate solid tissue (Gowrishankar and Weaver 2003, 2006; Esser et al. 2007, 2009). More recent examples of cell EP modeling encompass a variety of applications (Merla et al. 2011, 2012; Elia et al. 2010; Li and Lin 2011; Suzuki et al. 2011; Sadik et al. 2013; Kaner et al. 2013; Rems et al. 2013).

\section{Molecular Dynamics (MD) Electroporation Modeling}

Starting about a decade ago MD has also been applied to the formation of transient defects ("pores") in lipid bilayer membranes, due to both mechanical stress and to elevated transmembrane voltages (Tieleman et al. 2003; Tieleman 2004; Tarek 2005; Vernier et al. 2006b; Vernier and Ziegler 2007; Gurtovenko and Vattulainen 2007; Böckmann et al. 2008; Levine and Vernier 2010; Romeo et al. 2013; Bennett et al. 2014). Most MD modeling has not only emphasized electrical creation of pores in small planar membranes, but also treat vesicular and other "out of plane" structural rearrangements.

\section{Background for Present Model}

Our approach uses continuum modeling. It features simple to complex membrane geometries, fixed (passive), and dynamic (active; transmembrane voltage-responsive) properties. We assign each type of membrane a resting potential source, such that as pores are created, their combined conductance shunts the source, and the transmembrane voltage, $\Delta \phi_{\mathrm{m}}$, decreases. We also assign a complex, dynamic local membrane EP model that describes pore creation, evolution (expansion/contraction), and eventual stochastic destruction (Pastushenko et al. 1979; Levine and Vernier 2010; Smith et al. 2013).

Cell level models can be based on relatively simple single-closed curved membranes that represent the outer PM. More complex and realistic cell models include explicit intracellular membranes that represent organelles. With the exception of spherical models that include a concentric inner membrane, others populate the cell interior with one or more organelle membranes at asymmetric locations. Additional realism is found in the use of double membranes that represent the nuclear envelope or the inner and outer mitochondrial membranes (Gowrishankar et al. 2006; Esser et al. 2010; Gowrishankar et al. 2011).

\section{Models of Biological Systems Require Approximations}

Modeling is pursued to gain useful insights, including confirmation of expectations but also discovering new, unexpected behavior. We accept the view that a model is a hypothesis (Slepchenko et al. 2003), subject to testing and refutation, and can also lead to improvement of the hypothesis (see "Future Directions" section). Given the complexity of real cells and their membranes, we emphasize understanding model responses, related to some aspects of experiments, such as uptake or release of ions and molecules. The complexity of cell modeling also means that we must make approximations, particularly in the view of computational limitations.

\section{Importance of Electrodiffusive Solute Transport Rates}

The traditional emphasis of EP is introduction of solutes to the cytoplasm. Now EP is also of interest for removal of solutes from the cytoplasm. Obtaining samples for external analysis is one goal. Killing cells by accidental necrosis (Galluzzi et al. 2012) is another, the basis of irreversible electroporation (IRE) for nonthermal tumor ablation (Davalos et al. 2004; Miller et al. 2005; Rubinsky et al. 2008; Garcia et al. 2011; Golberg and Yarmush 2013). Release of important biomolecules and ions is basic to necrosis. 
Further, intrinsic apoptosis (Galluzzi et al. 2012) is the basis of nonthermal cell death by nanosecond electric field (nsPEF) (Beebe, et al. 2002; Nuccitelli et al. 2006; Garon et al. 2007; Nuccitelli et al. 2014). In this case, release of solutes from organelles such as the endoplasmic reticulum and mitochondria is important, but at the same time molecules needed for apoptosis should remain in the cytoplasm, a quantitative issue. Both uptake and release of solutes are therefore relevant to current research topics and applications in EP.

\section{Objective of This Paper}

Here we describe a cell model, an extension by Smith (Smith 2011) of the cylindrical version of the traditional Schwan passive cell model (Pauly and Schwan 1959). The simplest forms of the Schwan equation for the spherical and cylindrical cell membranes are given in "Appendix."

In the present model, local models for EP have been added, along with a resting potential source. Coupled electrical and poration behavior is used to predict the solute transport by electrodiffusion. Two charged solutes are considered, calcein (green fluorescence) and propidium (red fluorescence), as these fluorescent probes are often used in EP research.

We present the model's response to a relatively small, long pulse with $1.5 \mathrm{kV} / \mathrm{cm}$ strength and $100 \mu$ s duration that is typical of widely used pulses in cancer tumor treatments to deliver drugs such as bleomycin into cells (electrochemotherapy or ECT) (Poddevin et al. 1991; Dev et al. 2000; Gothelf et al. 2003; Breton and Mir 2012), and in IRE that ablates by causing accidental necrosis by nonthermal pulses alone (Davalos et al. 2004; Miller et al. 2005; Rubinsky et al. 2008; Garcia et al. 2011). Frandsen et al. describe a further extension, calcium EP (Frandsen et al. 2012).

In addition, we present model responses to a relatively large, short pulse of $40 \mathrm{kV} / \mathrm{cm}$ amplitude, and $10 \mathrm{~ns}$ duration (Silve et al. 2012). Pulses of this general type (submicrosecond, megavolt per $\mathrm{m}$; also nsPEF or nanosecond pulsed electric fields) are of more recent interest (Schoenbach et al. 2001; Müller et al. 2001; Beebe, et al. 2002; Craviso et al. 2012; Nuccitelli et al. 2006; Garon et al. 2007; Napotnik et al. 2012). Overall, the cell system model is complicated, as required to account for observed behavior in cell experiments (Smith 2011; Canatella et al. 2001; Puc et al. 2003).

\section{Methods}

We describe our methods in both general and specific terms, with subsections that identify major features. Realistic models are a worthwhile approach to creating hypotheses for cell EP, which involves a very large parameter space that cannot be realistically addressed by experiments alone (Weaver et al. 2012).

\section{Model Geometry}

While some prior continuum models have used toroidal pore geometry (Smith et al. 2004; Smith 2011) or even simple cylindrical geometry (Weaver and Chizmadzhev 1996), we now use a trapezoidal pore (Fig. 1a), which has a cylindrical pore interior. This region of a pore is often rate limiting. We use this interior cylinder with spreading resistance assigned outside, to approximate both a pore's vestibules and the nearby regions outside the membrane adjacent the entrance and exit to the pore. The trapezoidal pore geometry is created by rotating a trapezoid around an axis perpendicular to the local membrane plane (Smith 2011; Smith et al. 2013). Here $d_{\mathrm{m}} \approx 5 \mathrm{~nm}$ is the average cell membrane thickness (Kotnik and Miklavčič 2000). This is larger than most bilayer membranes because it includes $50 \%$ membrane proteins in the PM. The half membrane thickness, $d_{\mathrm{m}} / 2$ is the radius of the rotated circle of the toroidal pore, and $r_{\mathrm{p}}$ is the pore radius (size), which can vary significantly. The length of the inner, cylindrical pore interior is $d_{\mathrm{p}}$, here taken to be half the average membrane thickness for simplicity.

Figure $1 \mathrm{~b}$ shows the idealized (cylindrical) geometry of an elongated molecule near a pore. Cylindrical geometry is compatible with well-established engineering approximate descriptions of steric hindrance and also charged solute partitioning based on Born energy changes (Parsegian 1969, 1975; Chernomordik et al. 1987; Glaser et al. 1988; Kakorin and Neumann 2002).

Figure 1c shows the cell model geometry, with the PM interfaced between the light blue extracellular and dark blue intracellular regions, all within the simulation box, with idealized planar electrodes at the top and bottom. We use the simplest representation of a PM, a closed, curved cylindrical membrane that separates the intracellular region from the external medium. Figure 1d, e shows the full simulation box and the dense meshing near the PM. The cell radius is $r_{\mathrm{m}}=10 \mu \mathrm{m}$, with cell height is $h_{\mathrm{m}}=13.3 \mu \mathrm{m}$, so that the cell volume is the same as for a spherical cell with the same radius.

Figure 1f shows the pore energy landscape (Smith et al. 2013), similar to previous landscapes. The landscape is populated with pores via a creation rate that injects pores at $r_{\mathrm{p}}^{*}=0.65 \mathrm{~nm}$. Once within the landscape, pore radii change (expand and contract) in response to drift and diffusion contributions as the local slope of the landscape changes with time as the local $\Delta \phi_{\mathrm{m}}$ changes. Pores can leave the landscape if they reach a bin just to the left of $r_{\mathrm{p}}^{*}$, where stochastic destruction occurs. Both creation and destruction are 
(a)

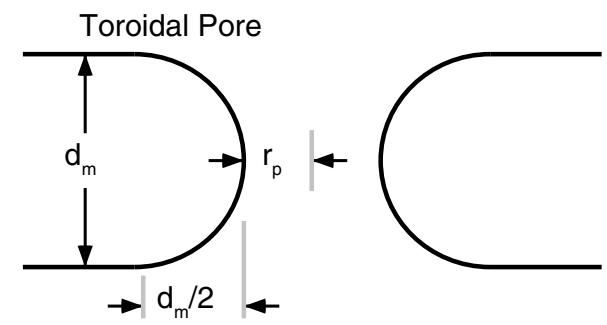

Trapezoidal Pore

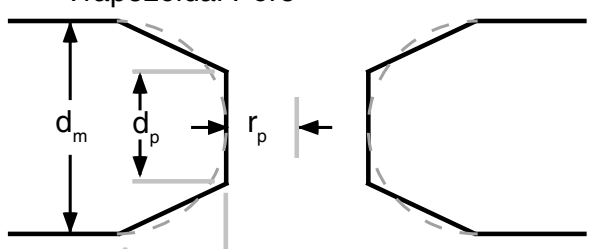

$\rightarrow d_{m} / 2$

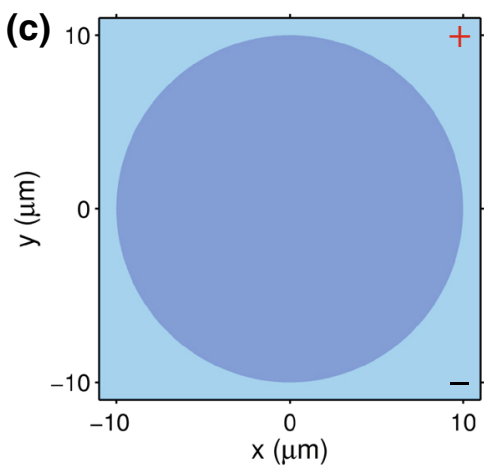

(d)
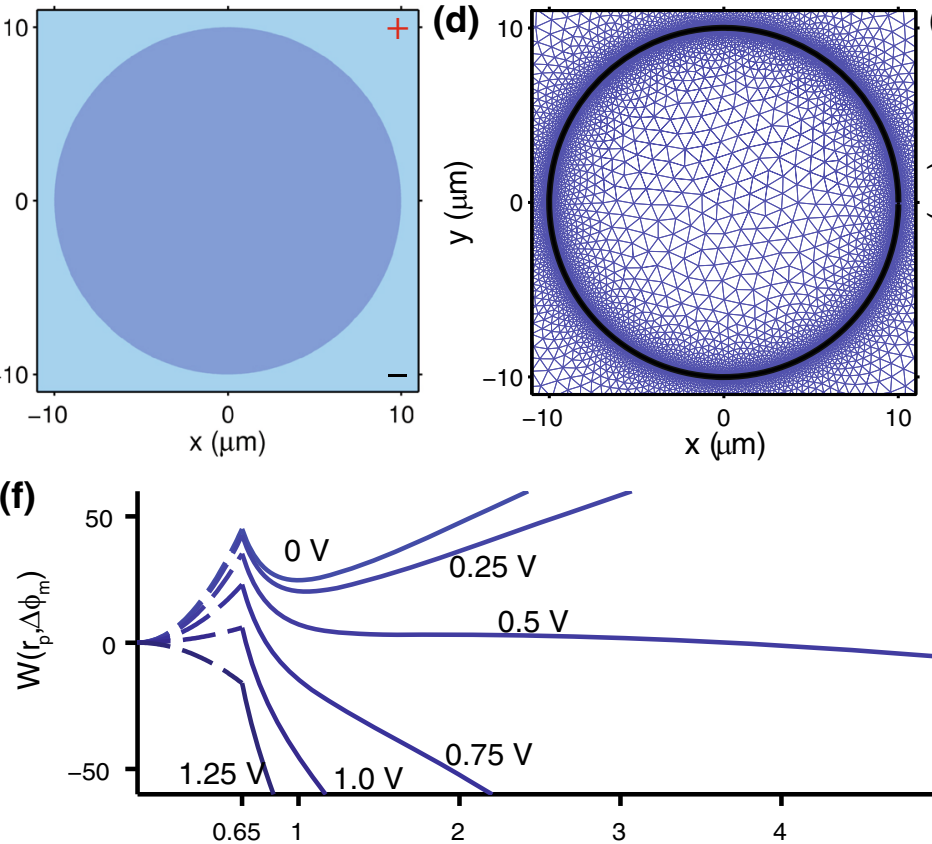

Fig. 1 a Top traditional toroidal pore geometry. Bottom present, simplified trapezoidal pore geometry. Here $d_{\mathrm{m}}$ is the average cell membrane thickness $(5 \mathrm{~nm}), r_{\mathrm{p}}$ is the pore radius at the most restrictive (narrow) interior pore region. b The trapezoidal pore approximation is used to calculate the electrodiffusive transport of a solute with length, $l_{\mathrm{s}}$ and radius, $r_{\mathrm{s}}$ through a pore of radius $r_{\mathrm{p}}$ and

governed by absolute rate equations, with creation having a strong and nonlinear dependence on the local $\Delta \phi_{\mathrm{m}}$.

\section{Creation and Evolution of Pore Populations During}

a Pulse

Dynamic pore populations (pore size distributions) are an underlying feature of cell membrane EP. An applied field (b)
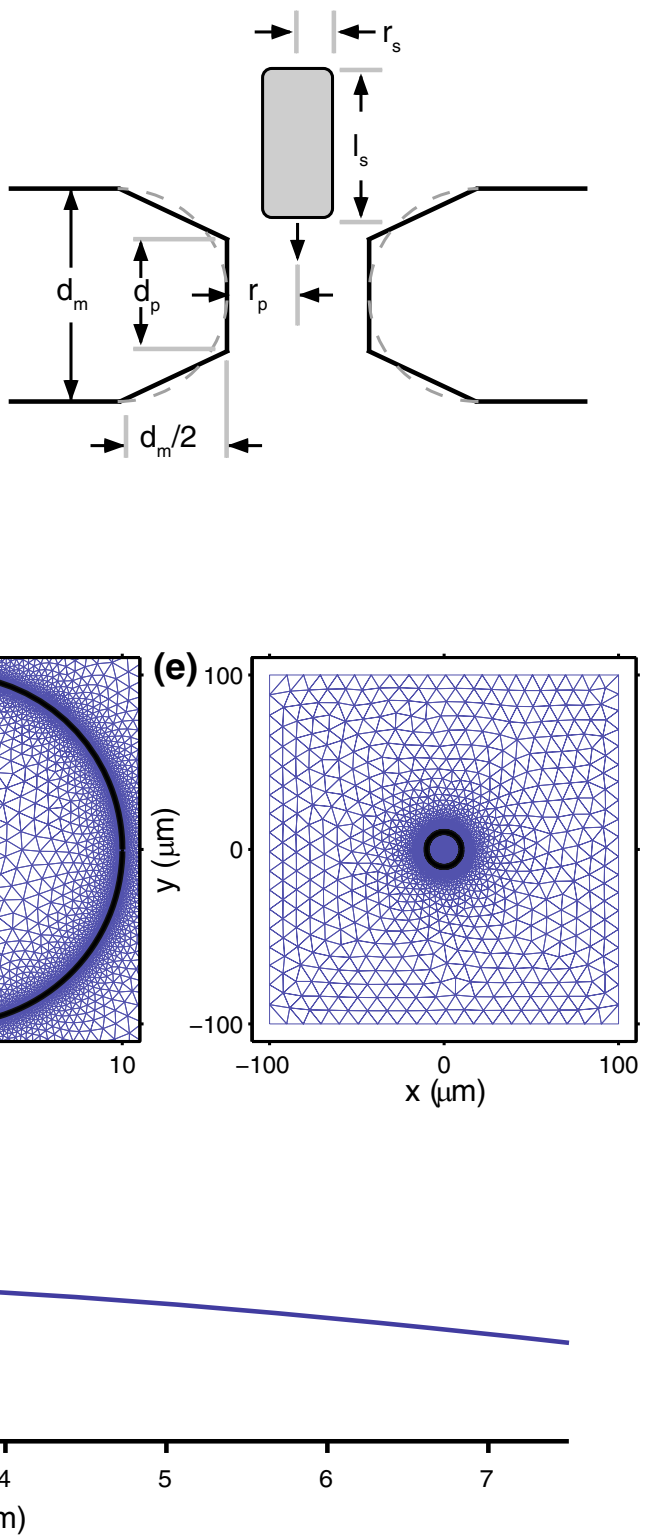

$r_{p}(n m)$

thickness $d_{\mathrm{p}}$. c The circular cell model consists of intracellular (dark blue) and extracellular (light blue) electrolyte, separated by a $5 \mathrm{~nm}$ thick membrane with $10 \mu \mathrm{m}$ radius. d Meshing for the circular cell model consisting of 600 node-pairs. e Same as d, but showing the entire $200 \mu \mathrm{m} \times 200 \mu \mathrm{m}$ system. f The total pore energy, $W\left(r_{\mathrm{p}}, \Delta \phi_{\mathrm{m}}\right)$, at increments of $0.25 \mathrm{~V}$ (Color figure online)

results in complex distributions of membrane fields, which change $\Delta \phi_{\mathrm{m}}(t)$ for each transmembrane node-pair. The pore populations have a complex dependence on the applied field which couples to the various local membrane sites by field amplification (Fig. SI-2, Supplemental Information). These populations are also the source of the inherent complexity of cell membrane EP. The populations are computed self-consistently with transmembrane charge transport through 

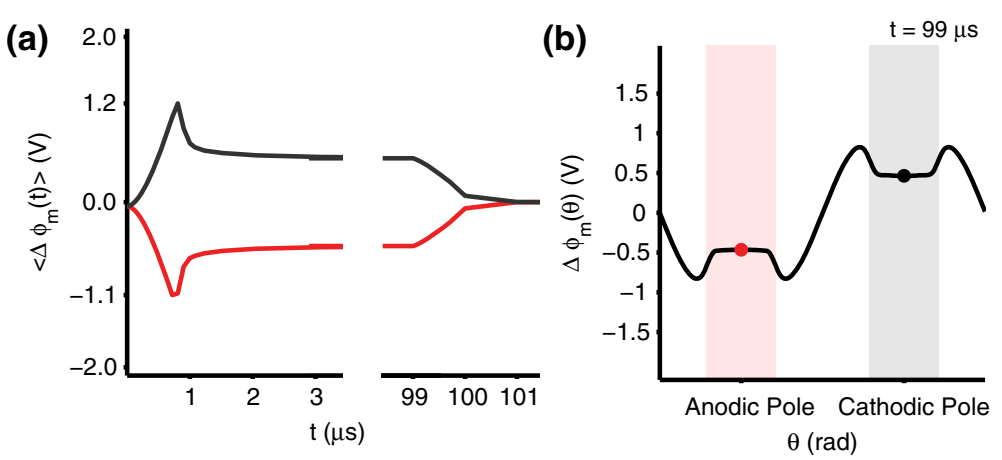

(c)

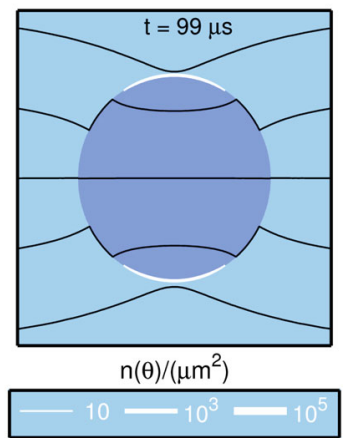

(d)

(e)
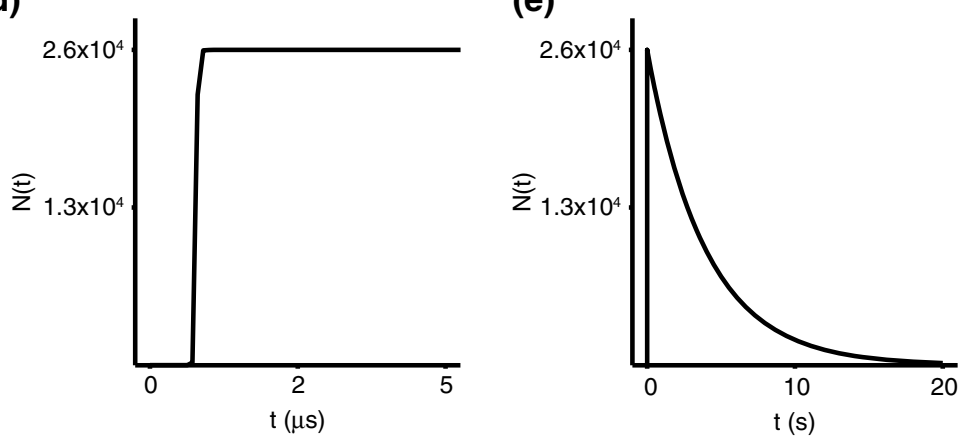

(f)

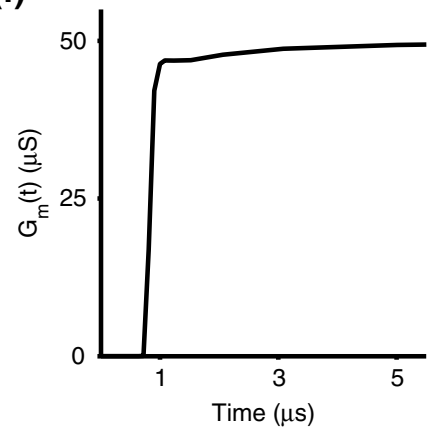

(g)

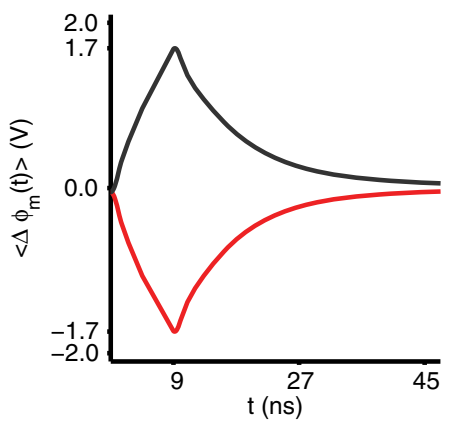

(h)

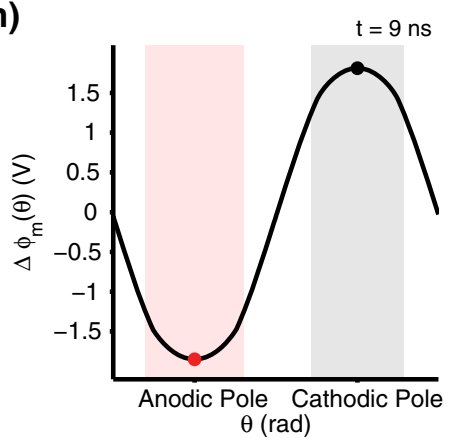

(i)

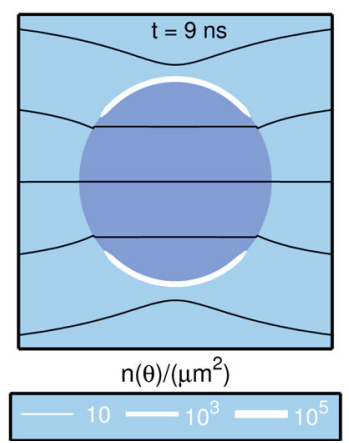

(j)

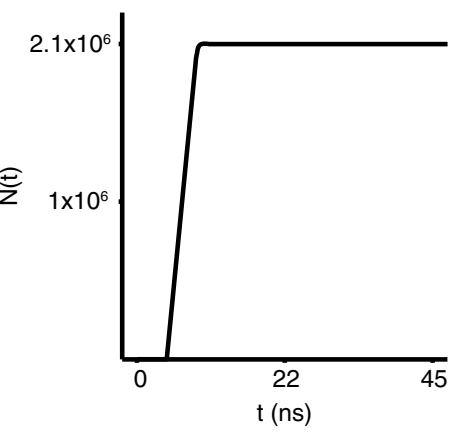

(k)
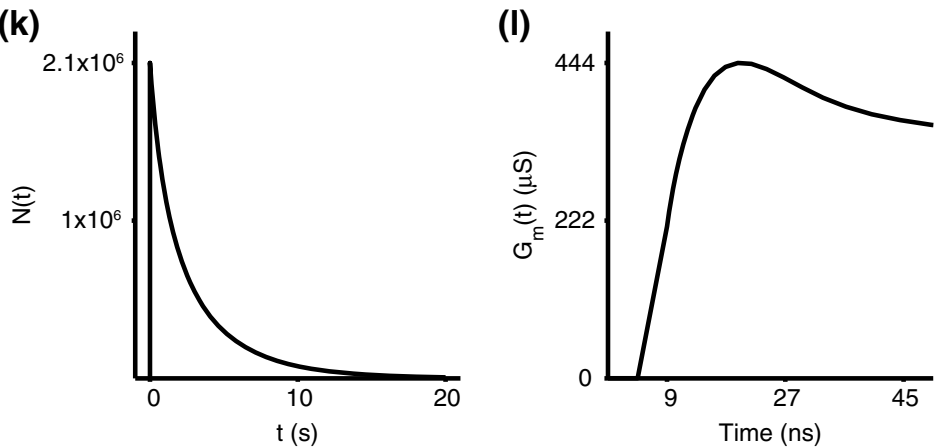

Fig. 2 Electrical and poration behavior for a $1.5 \mathrm{kV} / \mathrm{cm}, 100 \mu$ s pulse (a-f) and a $40 \mathrm{kV} / \mathrm{cm}, 10 \mathrm{~ns}$ pulse $(\mathbf{g}-\mathbf{I})$. Average transmembrane voltage, $\left\langle\Delta \phi_{\mathrm{m}}(t)\right\rangle$, is spatially averaged over the polar quadrants. Angular transmembrane voltage, $\Delta \phi_{\mathrm{m}}(\theta)$ at the end of pulse maximum shows the spatial heterogeneity in the electrical response across the membrane. Light red and black shaded regions represent electroporated regions of the membrane. Similarly, pore density, $n(\theta)$, at the end of pulse maximum shows the spatial extent of electroporation. The total number of pores, $N(t)$, rises after REB, and decreases exponentially after the pulse in accordance with pore lifetime, $\tau_{\mathrm{p}}=4 \mathrm{~s}$. Similarly, the total membrane conductance, $G_{\mathrm{m}}(t)$, increases with pore creation and expansion (Color figure online) 
several hundred different dynamic pore populations during a pulse. Examples of pore distributions on the anodic and cathodic sides of the cell are displayed in Fig. 3.

\section{Meshed Network Equivalent to Very Large Circuit}

The meshed transport network model (Fig. 1e) is generated by assigning local model to 600 membrane sites and the surrounding electrolyte. These interconnected elements can be regarded as a very large circuit that contains both linear, fixed components $(9,267$ nodes within intra- and extracellular aqueous regions), and highly nonlinear active components (transmembrane node-pairs). Each of the local areas associated with a transmembrane node-pair is regarded as a very small planar membrane patch endowed with a resting potential source and a complete dynamic EP model. Solutions of the circuit yield potentials at the many extraand intracellular nodes, which allow equipotentials to be computed and displayed throughout all or portions of the system at any time. The pairs of potentials at the transmembrane nodes allow the transmembrane voltage, $\Delta \phi_{\mathrm{m}}$, to be similarly computed. These are spatially averaged and displayed as a function of time (Fig. 2a, g).

\section{System Model has Passive and Active Components}

The model is constructed from established passive and active (nonlinear, hysteretic) components. These components range from simple conductive and dielectric (capacitive) models to a complete membrane EP model for a small planar membrane patch (Smith 2011). The most complicated local models are located at the membrane, here inserted between each of 600 node-pairs. These models account for changing pore energy landscapes and two rate equations (pore creation and destruction rates) that govern pore population evolution based on pore creation, expansion and contraction, and destruction. Dynamic pore behavior is computed at each transmembrane node-pair, based on the local $\Delta \phi_{\mathrm{m}}$ and the pore population. During a pulse the pore energy changes at each of the 600 node-pairs. This means that the pore energy gradients change, leading to changing drift and diffusion rates in pore radius space at each of these transmembrane sites (Smith et al. 2013). Overall, the interconnected system of local models is complex, needed to describe the many interactions that together define cell membrane EP. The different parameters and their values used in the system model are listed in Table 1.

Solute Transport by Electrodiffusion Within Bulk and Through Dynamic Pores

The important topic of specific ions and molecules (solutes) transport is addressed by electrodiffusion in the transport number approximation, which utilizes already-
Table 1 Model parameters (Smith et al. 2013)

\begin{tabular}{ll}
\hline Parameter & Value \\
\hline Cylindrical cell radius $\left(r_{\mathrm{m}}\right)$ & $10 \mu \mathrm{m}$ \\
Cylindrical cell height $\left(h_{\mathrm{m}}\right)$ & $13.3 \mu \mathrm{m}$ \\
Membrane resting potential $\left(\Delta \phi_{\mathrm{m}, \mathrm{rest}}\right)$ & $-50 \mathrm{mV}$ \\
Membrane thickness $\left(d_{\mathrm{m}}\right)$ & $5 \mathrm{~nm}$ \\
Radius of pore creation $\left(r_{\mathrm{p}}^{*}\right)$ & $0.65 \mathrm{~nm}$ \\
Pore radius at local energy minimum $\left(r_{\mathrm{p}, \mathrm{min}}\right)$ & $1 \mathrm{~nm}$ \\
Pore radius maximum $\left(r_{\mathrm{p}, \text { max }}\right)$ & $60 \mathrm{~nm}$ \\
Pore lifetime $\left(\tau_{\mathrm{p}}\right)$ & $4 \mathrm{~s}$ \\
Extracellular electrolyte conductivity $\left(\sigma_{\mathrm{ext}}\right)$ & $1.2 \mathrm{~S} / \mathrm{m}$ \\
Intracellular electrolyte conductivity $\left(\sigma_{\text {int }}\right)$ & $0.3 \mathrm{~S} / \mathrm{m}$ \\
Calcein charge number & -4 \\
Calcein radius $\left(r_{\text {cal }}\right)$ & $0.58 \mathrm{~nm}($ Smith 2011) \\
Calcein length $\left(l_{\text {cal }}\right)$ & $1.89 \mathrm{~nm}($ Smith 2011) \\
Calcein initial concentration $\left(\gamma_{\text {cal,ext }, 0}\right)$ & $1 \mathrm{mM}$ \\
Propidium charge number & $+2(\mathrm{Smith} 2011)$ \\
Propidium radius $\left(r_{\text {pro }}\right)$ & $0.69 \mathrm{~nm}$ (Smith 2011) \\
Propidium length $\left(l_{\text {pro }}\right)$ & $1.55 \mathrm{~nm}$ (Smith 2011) \\
Propidium initial concentration $\left(\gamma_{\text {pro,ext }, 0}\right)$ & $1 \mathrm{mM}$ \\
Temperature $(T)$ & $298.15 \mathrm{~K}$ \\
\hline &
\end{tabular}

determined electric fields to compute electrodiffusion (Smith 2011; Smith and Weaver 2012). Two types of regions are treated simultaneously, via interconnections as in circuit theory. The first case is bulk electrodiffusive transport within the extra- and intracellular media with electrodiffusion through the individual pores of different pore populations that are located between the transmembrane node-pairs. The detailed description of individual pore solute electrodiffusion includes partitioning into pores and sterically hindered movement through the interior pore region (Smith 2011). Solute transport through many complex dynamic pores in the membrane is both rate limiting and computationally nontrivial, and is intimately connected to and within the bulk aqueous regions.

Here we consider two fluorescent probe molecules used in EP research, calcein and propidium (the cation of dissociated propidium iodide, PI). Calcein $(623 \mathrm{Da})$ has $r_{\mathrm{cal}}=$ $0.58 \mathrm{~nm}$ and $l_{\text {cal }}=1.89 \mathrm{~nm}$ and a charge of -4 . Propidium (415 Da) has charge number $z=+2$, radius $r_{\text {pro }}=$ $0.69 \mathrm{~nm}$, and length $l_{\text {pro }}=1.55 \mathrm{~nm}$ (Smith 2011). The number of molecules within the cell is treated as free (dissolved). For simplicity, we do not represent the binding of propidium to intracellular nucleic acids (Sadik et al. 2013).

\section{Idealized and Actual Pulse Waveforms}

To aid interpretation of results, we used idealized trapezoidal pulses, with linear ramps that define the rise and fall 
times, and a flat peak field strength (Fig. SI-1). The model can also be used with digitized experimental waveforms (Gowrishankar et al. 2006, 2011).

\section{Conventional Electroporation Pulse}

For mammalian cells most pulses have strengths of 0.1 to $\sim 10 \mathrm{kV} / \mathrm{cm}$ and durations longer than a microsecond. As an example, we use an idealized trapezoidal pulse with strength $1.5 \mathrm{kV} / \mathrm{cm}$, duration $100 \mu \mathrm{s}$, and a rise/fall time of $1 \mu \mathrm{s}$, an approximation to a widely used pulse. Pulse trains that employ eight $100 \mu$ s pulses were first used in research (Mir et al. 1988), and subsequently in electrochemotherapy (ECT) at a repetition rate of $1 \mathrm{~Hz}$ (Poddevin et al. 1991; Dev et al. 2000; Miklavčič et al. 2005), in IRE (Davalos et al. 2005; Miller et al. 2005; Rubinsky et al. 2008; Rubinsky 2010; Garcia et al. 2011; Breton and Mir 2012; Kennedy et al. 2014) and in calcium electroporation (Frandsen et al. 2012).

\section{Submicrosecond, Megavolt per Meter Pulse for Supra- Electroporation}

The second pulse example is also an idealized, trapezoidal pulse, with strength $40 \mathrm{kV} / \mathrm{cm}$, duration $10 \mathrm{~ns}$, and linear ramps that define the $1 \mathrm{~ns}$ rise and fall times. Although terminology varies, a common designation is nsPEF (nanosecond pulsed electric field), indicating pulses with durations less than $1 \mu \mathrm{s}$. Such pulses have received considerable attention since 2001, when two papers appeared several months apart (Schoenbach et al. 2001; Müller et al. 2001). These pulses are close to the conditions studied by MD (Tieleman et al. 2003; Vernier et al. 2006b; Vernier and Ziegler 2007; Levine and Vernier 2010; Fernández et al. 2012; Romeo et al. 2013; Delemotte and Tarek 2012), and involve pore densities expected for supra-EP (Stewart et al. 2004; Gowrishankar et al. 2006; Esser et al. 2009). Potential applications include nonthermal cell death by apoptosis (Beebe, et al. 2002; Beebe, et al. 2003; Nuccitelli et al. 2006; Garon et al. 2007; Nuccitelli et al. 2014), and a variety of other intracellular effects. Even the introduction of bleomycin is reported for nsPEF (Silve et al. 2012).

\section{Results and Discussion}

Electrical and Poration Behavior

Figure 2 shows examples of coupled electrical and poration behavior. Movement of small ions through pores and toward or away from the membrane is used to compute currents, fields, and transmembrane voltages, with the latter two quantities emphasized here. Charged solute transport contributes negligibly to electrical behavior. Accordingly, solute transport by electrodiffusion is computed using the electric fields determined solely by small, ubiquitous ions.

\section{Transmembrane Voltage Temporal Behavior}

Electroporation is characterized by electrical creation of transient aqueous pores, usually described by a rate equation that is strongly dependent on the transmembrane voltage, $\Delta \phi_{\mathrm{m}}$ (Weaver and Chizmadzhev 1996; Neu and Krassowska 1999; Smith 2011; Smith et al. 2013). At one extreme, a pore can be created in about one nanosecond for $\Delta \phi_{\mathrm{m}} \approx 1.2 \mathrm{~V}$ in a planar membrane patch (Vasilkoski et al. 2006), with similar times reported by MD studies using pure lipid bilayers (Gurtovenko and Vattulainen 2007). For longer times much smaller voltages are sufficient (Melikov et al. 2001). Cell models show behavior consistent with similar rate equations (DeBruin and Krassowska 1999a, b; Stewart et al. 2004; Krassowska and Filev 2007) and include the present model (Smith 2011; Smith et al. 2013).

The transmembrane voltage, $\Delta \phi_{\mathrm{m}}$, is a key quantity for pore creation, and for dynamic pore behavior involving expansion and contraction, with the latter important to pores shrinking to a small enough size that they are destroyed by thermal fluctuations (Pastushenko et al. 1979; Levine and Vernier 2010; Smith et al. 2013). Figure 2a, g shows $\Delta \phi_{\mathrm{m}}$ averaged over the cathodic and anodic quadrants for the two pulses, with $\Delta \phi_{\mathrm{m}}(\mathrm{t})$ behavior that is quite different due to the very different durations and rise/fall times.

Figure 2a shows the response to the conventional EP pulse $(1.5 \mathrm{kV} / \mathrm{cm}, 100 \mu \mathrm{s})$. The display emphasizes the transient behavior at the beginning of the pulse (first $3 \mu \mathrm{s}$ ) and at the pulse end (from 99 to $101 \mu \mathrm{s}$ ). The membrane charges passively until $\mathrm{t}=0.7 \mu \mathrm{s}$, when the rise in $\Delta \phi_{m}$ is interrupted by REB (Benz et al. 1979; Barnett and Weaver 1991). This transmembrane voltage peak of $1.2 \mathrm{~V}$ is associated with a burst of pore creation. The accompanying large conductance increase interrupts the $\Delta \phi_{\mathrm{m}}$ rise, and forces a change in direction such that $\Delta \phi_{\mathrm{m}}$ quickly decreases, even though the applied field is still increasing. Within a microsecond after the REB peak the rate of $\Delta \phi_{\mathrm{m}}$ decreases slowly, and reaches a quasi-plateau of about $0.5 \mathrm{~V}$.

Figure $2 \mathrm{~b}, \mathrm{k}$ shows the angular profiles of $\Delta \phi_{\mathrm{m}}$ at the end of the maximum values of the two pulses. For the $100 \mu$ s pulse this is $99 \mu$ s, which is much longer than five charging times $\left(\tau_{\mathrm{m}}=370 \mathrm{~ns}\right)$. But the idealized $10 \mathrm{~ns}$ pulse peak value ends at $9 \mathrm{~ns}$, which is much shorter than $\tau_{\mathrm{m}}$. This means that pore creation begins rapidly, at about $5 \mathrm{~ns}$ (Fig. 2j). At $\Delta \phi_{\mathrm{m}} \approx 1.7 \mathrm{~V}$ (Fig. $2 \mathrm{~g}$ ) pores are made rapidly, even though the membrane never charges fully. Instead, a large local conductance exists when the pulse maximum value ends at $9 \mathrm{~ns}$, and $\Delta \phi_{\mathrm{m}}$ begins to relatively slowly decrease to zero over about $40 \mathrm{~ns}$ (Fig. $2 \mathrm{~g}$ ). 

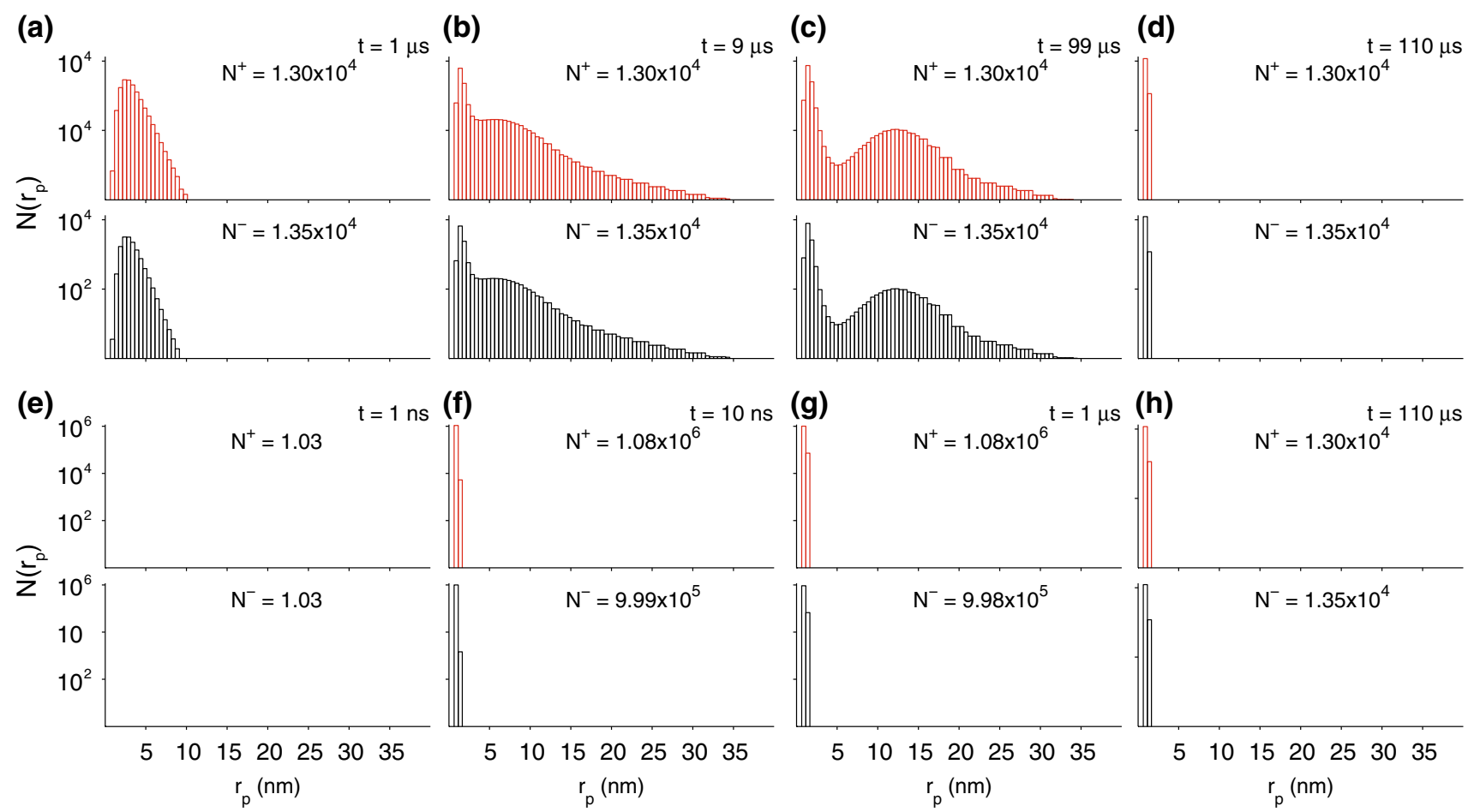

Fig. 3 Pore histograms show the distribution of pore radii, $N\left(r_{\mathrm{p}}\right)$, at various times in response to the $1.5 \mathrm{kV} / \mathrm{cm}, 100 \mu \mathrm{s}$ pulse $(\mathbf{a}-\mathbf{d})$ and the $40 \mathrm{kV} / \mathrm{cm}, 10 \mathrm{~ns}$ pulse $(\mathbf{e}-\mathbf{h})$. The maximum pore radius

$r_{\max }=60 \mathrm{~nm}$, but the display has been truncated to $40 \mathrm{~nm}$ in consideration of the maximum pore radii observed

\section{Transmembrane Voltage Angular Profiles}

Flattening of the transmembrane voltage profile around a cell is a classic indicator of EP, usually shown as the angular profile of $\Delta \phi_{\mathrm{m}}$. Flattening is observed experimentally (Kinosita et al. 1988; Frey et al. 2006; Flickinger et al. 2010), and is also exhibited by cell membrane models and has been found in cell model responses for both spherical (DeBruin and Krassowska 1999a; Smith and Weaver 2008; Talele et al. 2010; Sadik et al. 2013), and cylindrical membranes (Stewart et al. 2004; Gowrishankar et al. 2006; Smith et al. 2013). Figure 2b, h shows the model's angular response for the two pulses. In addition to profile flattening around the poles, the local peaks in Fig. $2 \mathrm{~b}$ indicate transition regions that separate porated (shaded regions) from unporated membrane (DeBruin and Krassowska 1999a; Krassowska and Filev 2007; Esser et al. 2010).

\section{Pore Densities}

The model's poration response is shown as pore density plots (Fig. 2c, i) for both pulses, and shows where pores are present, independent of their size at the time indicated. Here the pore density (number of pores per square $\mu \mathrm{m}$ ) is

indicated by white bands superposed on the cell membrane. The width of the white bands is proportional to pore density. Figure $2 \mathrm{c}$ shows two thin white bands that occupy about a third of the membrane, with $2.7 \times 10^{4}$ pores (Fig. $3 \mathrm{a}-\mathrm{d}$ ) created by a $1.5 \mathrm{kV} / \mathrm{cm}, 100 \mu$ s pulse. The time is the end of the pulse peak, $99 \mu$ s, when pores have evolved to form two subpopulations (Fig. 3c). Similarly, Fig. 2i shows two broad white bands that occupy more of the membrane, with $2.1 \times 10^{6}$ pores (Fig. $3 \mathrm{f}, \mathrm{h}$ ) created by a $40 \mathrm{kV} / \mathrm{cm}$, $10 \mathrm{~ns}$ pulse. The time is also at the end of the pulse peak, 9 ns. For this much larger and shorter pulse, there is very little pore expansion (Fig. $3 \mathrm{f}-\mathrm{h}$ ). All the pores are small, but there are about 100-fold more pores.

\section{Total Pore Number}

The total number of pores in the cell membrane, $N$, is another partial indicator of EP. A general expectation is that more pores provide greater transport capability for small ions that dominate electrical behavior and for small solutes. According to the model, $N(t)$ rapidly increases during a pulse, but after a pulse $N(t)$ decreases exponentially with a 4 s pore lifetime (recovery or resealing time). This general behavior is a consequence of the importance of electrical interactions during a pulse. However, membrane depolarization results for most pulses, resulting in 
$\Delta \phi_{\mathrm{m}} \approx 0$ post pulse, with pore destruction proceeding spontaneously, due to local fluctuations. This is consistent with MD simulations, but MD lifetimes are much shorter, for reasons not yet understood.

For the conventional EP pulse, Fig. 2d shows the initial increase in total pores, which occurs in less than a microsecond, well before the pulse has reached its peak value. On a much longer time scale Fig. 2e shows $N$ both during the pulse and for five pore lifetimes (20 s total) of stochastic pore decay post pulse. During essentially all of the post pulse decay, there are only thermalized pore distributions (Fig. 3d, h). The shape of the thermalized distribution is narrow, collapsed to only small pores. This narrow distribution is on average retained, but the magnitude, $N(t)$, of the population decays exponentially (Fig. 2e, $\mathrm{k})$.

The response to the nsPEF pulse (Fig. 2j) also shows a rapid initial increase in total pores. In this case, the pulse ends (at $9 \mathrm{ns)}$ before REB occurs, but already about two million pores have appeared, all small (Fig. $3 \mathrm{f}-\mathrm{h}$ ). Both the small pores and their large number are features of supra-EP (Stewart et al. 2004; Smith and Weaver 2008; Joshi and Schoenbach 2010), which is also predicted to porate many organelles. The longer time scale result again shows a sharp increase in $N$, followed by the same exponential decay (Fig. 2k), but the magnitude of $N$ is two orders of magnitude larger.

\section{Membrane Conductance}

The cell membrane conductance, $G_{\mathrm{m}}$, is closely related to $N$ for the simple reason that the predominant small ions $\left(\mathrm{Na}^{+}, \mathrm{K}^{+}\right.$and $\left.\mathrm{Cl}^{-}\right)$are so small that they easily pass through even small pores. A $\Delta \phi_{\mathrm{m}}$-dependent partitioning of these ions into pores is expected which gives $G_{\mathrm{m}}$ an obvious dependence on $N$, but also on $\Delta \phi_{\mathrm{m}}$ (Chernomordik et al. 1987; Glaser et al. 1988; Kakorin and Neumann 2002). The total conductance is important, because almost independent of how it is spatially distributed a large $G_{\mathrm{m}}$ is effective in shunting the resting potential source (DeBruin and Krassowska 1999a; Smith et al. 2013). For this reason most porating pulses lead to post pulse membrane depolarization. The shunting of that source by the 1,000 or more pores created by most pulses leads to $\Delta \phi_{\mathrm{m}} \approx$ zero after a pulse.

Pore Population Life Cycles: Creation, Evolution and Destruction

Pore populations, also termed pore size distributions, yield more detailed insights beyond $N(t)$. Here we show examples of pore populations created by the two pulses. For both pulses, the pre-pulse condition is an average of two pores total, one on each side (Fig. 3e). The most interesting phase of a population life cycle is from the beginning of additional pore creation by an applied pulse, to the time where the pulse ends. After that there is a general pore contraction that leads to a thermalized pore population in about $10 \mu \mathrm{s}$ (not shown). The pore contraction depends on discharge of the membrane, such that $\Delta \phi_{\mathrm{m}}$ goes to zero somewhat after the pulse end (Fig. 2a, g).

The thermalized populations have the same shape on average (Fig. 3d, g, h). Such distributions arise from thermal equilibrium and the shape of the pore energy, $W$, around the minimum (the $0 \mathrm{~V}$ curve of (Fig. 1f)) (Neu and Krassowska 1999; Smith 2011; Smith et al. 2013). This collapsed, post pulse population then decays with a relatively long pore lifetime (here $4 \mathrm{~s}$ ) that is believed to depend on cell membrane composition and temperature. While these are common features before and after a porating pulse, the detailed population behavior is quite different for the two pulses. Long porating pulses lead to populations with a wide range of sizes (Fig. 3a-d), whereas short pulses do not (Fig. 3e, h). According to essentially all theories and MD simulations, once created, pores tend to evolve to complex size distributions, while $\Delta \phi_{\mathrm{m}}$ is elevated, and only vanish when they contract to a minimum size. A detailed discussion of pore dynamics follows.

\section{Basal Rate and Spontaneous Pore Creation}

The basal rate of pore creation is not zero at the resting potential (here $-50 \mathrm{mV}$ ). The result of a field pulse is to increase an existing rate, not to start a new process. Accordingly, even a fully depolarized membrane $\left(\Delta \phi_{\mathrm{m}}=0\right)$ has a very small but nonzero spontaneous pore creation rate, due to thermal fluctuations. Present theories and models use an absolute rate equation that is very nonlinear in $\Delta \phi_{\mathrm{m}}$, which results in large numbers $(>1,000)$ of pores for typical pulse strengths and durations. From this it also follows that there is no critical field or transmembrane voltage. Instead, both an elevated $\Delta \phi_{\mathrm{m}}$ and a finite time are involved in producing a detectable number of pores. Accordingly, the model's pre-EP condition of $\bar{N}>0$ is consistent with the idea that nonionizing fields only modulate ongoing processes in biological systems (Vasilkoski et al. 2006).

\section{Local, Spontaneous Pore Destruction}

In the present model, stochastic pore destruction occurs at $r_{\mathrm{p}}^{*}=0.65 \mathrm{~nm}$ (Fig. 1f). Most pores are destroyed after a pulse, removed stochastically from thermalized distributions at $\Delta \phi_{\mathrm{m}} \approx 0$ (Fig. $3 \mathrm{~d}, \mathrm{~g}, \mathrm{~h}$ ). In pore radius space this 
means that some pore radii move over the landscape (Fig. 1f) on the $0 \mathrm{~V}$ curve to reach $r_{\mathrm{p}}^{*}$, where they become candidates for random removal by local fluctuations, according to an absolute rate equation. Pores expand and contract within the changing $\left(\Delta \phi_{\mathrm{m}}\right.$-dependent) landscape by both drift (force) and diffusion (random walk). Mathematically, this is analogous to electrodiffusion, and is solved using the descretized method employed for solute electrodiffusion (Smith and Weaver 2012).

One interpretation is that "pore lifetime" may be explained by involving two distinguishable processes: (1) the analog of electrodiffusion within the landscape, and (2) stochastic destruction that is local, here at slightly below $r_{\mathrm{p}}^{*}$. That is, pore loss is due to both the local stochastic destruction rate and transport in $r_{\mathrm{p}}$ space to the destruction site slightly below, $r_{\mathrm{p}}^{*}$. In one case, the destruction process could be mostly governed by diffusion, analogous to diffusion-limited chemical reactions. Alternatively, MD studies may show that the final stages of contraction and destruction comprise a single, multi-molecule event.

\section{Supra-Electroporation Pore Populations by nsPEF}

Because of its relative simplicity, we begin a brief detailed discussion of the pore population examples by considering the $40 \mathrm{kV} / \mathrm{cm}, 10 \mathrm{~ns}$ pulse response (Fig. 3e-h). The pores are small and the population behavior is limited. Figure $3 \mathrm{e}$ is early ( $t=1 \mathrm{~ns}$ ), and can be regarded as the pre-EP condition (Fig. 2j). Both the top (anodic) and bottom (cathodic) display the total pore number summed over the respective cell side. In Fig. 3e this is 1.03 pores on each side. This is the equilibrium number of spontaneous pores generated by equilibrium between two different, nonzero rates: spontaneous pore creation and stochastic pore destruction. The occasional spontaneous pore creation is followed by random destruction with a mean lifetime of $4 \mathrm{~s}$. Together, these rates of creation and destruction are the basis of $N^{+}$and $N^{-}=1.03$ on the anodic and cathodic sides. The average total number of pores is thus $\bar{N} \approx 2$, a small value which guarantees major fluctuations.

The nsPEF example shows that many pores exist at $10 \mathrm{~ns}$, when the field pulse drops to zero. $\Delta \phi_{\mathrm{m}}$ peaks at a magnitude of about $1.7 \mathrm{~V}$ on both the cathodic and anodic sides, at $9 \mathrm{~ns}$, the end of the field pulse maximum. This abrupt interruption of membrane charging nevertheless results in many pores, with slightly different numbers on the anodic $\left(\mathrm{N}^{+}=1.08 \times 10^{6}\right.$ pores $)$ and cathodic sides $\left(\mathrm{N}^{-}=9.99 \times 10^{5}\right.$ pores), so the total pore number is $\mathrm{N}=2.08 \times 10^{6}$. Here we show three significant figures, not because the model is that precise, but to make comparisons easier.
The main point of Fig. $3 \mathrm{f}-\mathrm{h}$ is that there are many pores, all small due to the short pulse duration, only during which pores can expand beyond $r_{\min }$. In addition, the highly nonlinear dependence of pore creation on $\Delta \phi_{\mathrm{m}}$ leads to many pores. The resulting large membrane conductance holds down $\Delta \phi_{\mathrm{m}}$ (Frey et al. 2006), to values far less than predicted for passive (nonporated) membranes (Stewart et al. 2004; Kotnik and Miklavčič 2006). Figure 3f-h shows narrow pore size distributions as expected for supra-EP (Stewart et al. 2004; Smith and Weaver 2008), which is a logical consequence of the model's construction (Methods), and are consistent with experimental electrical behavior (Frey et al. 2006) and observed fluorescent probe uptake (Vernier et al. 2006a; Pakhomov et al. 2007a, b, 2007a) and also bleomycin (Silve et al. 2012). Existing experimental and MD modeling results are consistent with the present model.

At $10 \mathrm{~ns}$ (pulse end), the pore population is represented by histograms with only two occupied bins ( $0.5 \mathrm{~nm}$ width), with a total of about $2.1 \times 10^{6}$ pores (Fig. $3 \mathrm{f}$ ). There is a slight asymmetry, with about $8 \%$ more pores on the anodic than cathodic side. There are also about 1,000-fold fewer pores in the larger bin. At $\mathrm{t}=1 \mu \mathrm{s}$ (Fig. 3g) the pulse has long ended, and the population has relaxed into the thermalized post pulse distribution (Fig. 3h), which but for the total pore number has the same distribution shape as Fig. 3d. A comparison of the populations in Fig. 3d, g, h shows the same pore size distribution shape, which means that each of these populations is in thermal equilibrium around $r_{\mathrm{p}, \min }$ for $0 \mathrm{~V}$ in the landscape of Fig. If (Neu and Krassowska 1999; Smith 2011; Smith et al. 2013). The similar shape of these thermalized distributions occurs because $\Delta \phi_{\mathrm{m}}=0$, and for our model only the $0 \mathrm{~V}$ curve of the landscape matters. The properties of such post pulse distributions are important to the post pulse diffusion of moderate size solutes.

\section{Conventional Electroporation Pore Populations}

Figure $3 \mathrm{a}-\mathrm{d}$ shows the more complex pore population behavior of the longer $1.5 \mathrm{kV} / \mathrm{cm}, 100 \mu$ s conventional EP pulse. Pores are created early in the pulse, and subsequent interactions within the model allow the emergence of a subpopulation of large pores. Emergence of such subpopulations has now been reported for three related cell EP models (Krassowska and Filev 2007; Esser et al. 2010; Smith et al. 2013). The time for emergence appears to be several microseconds. Pore creation essentially ceases (Fig. 2d) near the REB peak at $\sim 0.7 \mu \mathrm{s}$, with average $\Delta \phi_{\mathrm{m}}$ falling to a plateau of about $0.5 \mathrm{~V}$ (Fig. 2a). For this transmembrane voltage insignificant additional pore creation occurs on a $100 \mu$ s time scale.

When the pulse reaches its peak value at $1 \mu \mathrm{s}$, the distribution of pore radii has already broadened (Fig. 3a). The 
anodic distribution extends to $10 \mathrm{~nm}$ radius pores, and the cathodic population to slightly less at $9 \mathrm{~nm}$. At $9 \mu \mathrm{s}$, about $10 \%$ of the pulse duration, the pore size distribution has become more complex, with Fig. 3b showing the pore distribution beginning to divide into two subpopulations. Both the anodic and cathodic populations have become complex. There is a large peak at $\sim 1.5 \mathrm{~nm}$, and a barely discernible local maximum at $\sim 8 \mathrm{~nm}$ for both the anodic and cathodic sides. The pore size distribution at $1 \mu \mathrm{s}$ is slightly after the REB peak, when pore creation is complete (Fig. 2a, d). The total pore number is then $N=2.7 \times 10^{4}$, unchanged as the population evolves during the pulse (Fig. $3 \mathrm{a}-\mathrm{c})$. Some asymmetry occurs due to the resting potential source, with somewhat more pores on the cathodic (black histogram) than anodic (red histogram) side. The largest pores $(\sim 11 \mathrm{~nm}$ radius) appear on the anodic side, while the cathodic side has a maximum pore size of $\sim 9 \mathrm{~nm}$.

At $99 \mu \mathrm{s}$, the end of the pulse maximum, two distinct subpopulations have emerged on each side of the cell (Fig. 3c), but the number of pores on each side has not changed. This is consistent with insignificant pore creation for $\Delta \phi_{\mathrm{m}} \approx 0.5 \mathrm{~V}$ (Fig. 2a) on this time scale. Instead pore expansion and contraction occur, due to gradients within the pore energy landscape (Fig. 1f) (Smith et al. 2013). Finally, well after the pulse end at $110 \mu$ s the population has collapsed to form a thermalized pore distribution. This occurs at $10 \mu$ s after the pulse end (Fig. 3d). When the field pulse reaches zero approximately ten microseconds are needed for the cell membrane to discharge and for the pore populations to collapse by individual pore contraction until the thermalized distribution is achieved. With the exception of scaling by total pore number, $N$, the distributions in Fig. 3d, h are the same.

Overall, Fig. 3 illustrates a general feature of the model's response. Complex pore populations can develop during a pulse, but after the pulse the membrane conductance shunts the resting potential source, and the membrane is depolarized $\left(\Delta \phi_{\mathrm{m}} \approx 0\right)$. In pore radius space pores contract toward $r_{\mathrm{p}, \min } \approx 1 \mathrm{~nm}$, by drift, due to the slope in $\mathrm{W}$ at $\Delta \phi_{\mathrm{m}}=0$ (Fig. 1f). This collapse into a quasi-equilibrium distribution takes about $10 \mu \mathrm{s}$. If the scaling factor of $N$ is taken into account, all of the post pulse thermalized populations are the same, within fluctuations. Depending on pore lifetime (resealing or recovery time), post pulse solute transport occurs only through this type of pore distribution. The initial number of post pulse pores, and their lifetime, determines post pulse transport.

\section{Electrodiffusion of Solutes Within the System Model}

The model describes transport of ions and molecules by a descretized representation of electrodiffusion (Smith and Weaver 2012). Figures 4, 5 give examples of solute concentration distributions at different times for calcein (green) and propidium (red) for the conventional and supra-EP pulses, respectively. In both cases, the initial extracellular concentration is $1 \mathrm{mM}$. During a pulse, the drift contribution usually dominates. After a pulse the membrane is quickly depolarized and diffusion dominates. For a solute to enter a pore its radius $r_{\mathrm{s}}$ should be less than $r_{\mathrm{p}}$, the radius of the restrictive pore interior. This leads to a "soft cutoff" for transport as a function of size. It should also be energetically favorable for a charged solute to enter, which in the model involves a partition coefficient estimate based on electrostatic energy (Born energy) (Parsegian 1969, 1975). Post pulse diffusion should therefore favor propidium over calcein, in agreement with the model (Figs. 4, 5). Inside the pore solute movement is sterically hindered (Smith 2011).

To understand post pulse transport, a basic question is: What size and charge solutes pass through the thermalized distributions? We address this by providing a known amount of a particular solute within the extracellular region, and then applying a pulse. We know that small, biologically important ions readily pass through the decaying pore population. Water and small sugars can enter and diffuse through these pores, as can charged biologically active solutes such as ATP (507 Da, mainly charge number $z=-4$ and -3 ) (Smith 2011). Many membrane integrity probes can also pass through these pores, based on the cylindrical representation of their size (solute radius, $r_{\mathrm{s}}$, is most important; charge number also affects partitioning into a pore) (Smith 2011). Colorimetric and fluorescent dyes are already widely used in cell membrane electroporation research. Descriptions of transport of two medium size charged probes, calcein and propidium, are shown in Figs. 4, 5, 6, 7.

\section{Calcein Uptake for the $1.5 \mathrm{kV} / \mathrm{cm}, 100 \mu \mathrm{s}$ Pulse}

Figure $4 \mathrm{a}-\mathrm{d}$ shows calcein concentration (spatially distributed green) and the number of internalized calcein molecules (white number inside cell) at the indicated times for the $1.5 \mathrm{kV} / \mathrm{cm}, 100 \mu \mathrm{s}$ pulse. The difference in intracellular calcein numbers between Fig. 4a (pulse start; $t=$ $0 \mathrm{~s}$ ) and Fig. 4a (pulse end; $100 \mu \mathrm{s}$ ) shows that electrodiffusive drift dominates, with $3.2 \times 10^{7}$ molecules internalized at $100 \mu$ s. There is only $\sim 3 \%$ additional transport during a post pulse interval of five pore lifetimes $(20 \mathrm{~s}$; Fig. $4 \mathrm{~d})$ with $3.3 \times 10^{7}$ molecules. This is consistent with inhibition of calcein partitioning into the pore interior, due to its larger average charge number.

The intracellular green color distribution changes significantly between Fig. 4a, b. At the pulse start the intracellular space is black, indicating concentration, 

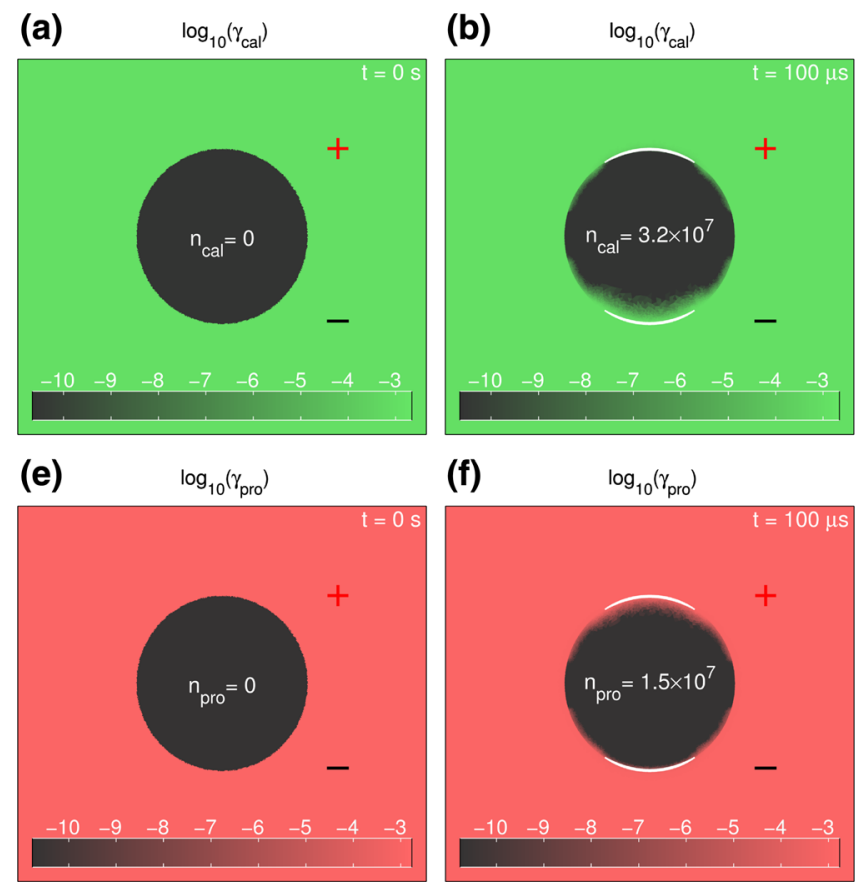

Fig. 4 The logarithm of calcein and propidium concentrations, $\gamma_{\text {cal }}$ and $\gamma_{\text {pro }}$, is shown at $t=0 \mathrm{~s}(\mathbf{a}, \mathbf{e}), \mathrm{t}=100 \mu \mathrm{s}(\mathbf{b}, \mathbf{f}), t=10 \mathrm{~ms}(\mathbf{c}, \mathbf{g})$, and $t=20 \mathrm{~s}(\mathbf{d}, \mathbf{h})$ for the $1.5 \mathrm{kV} / \mathrm{cm}, 100 \mu \mathrm{s}$ pulse. The total intracellular amounts of each solute are displayed inside the cell. Red ' + ' and black '-' indicate the anodic and cathodic sides of the system, respectively. The majority of calcein internalization is
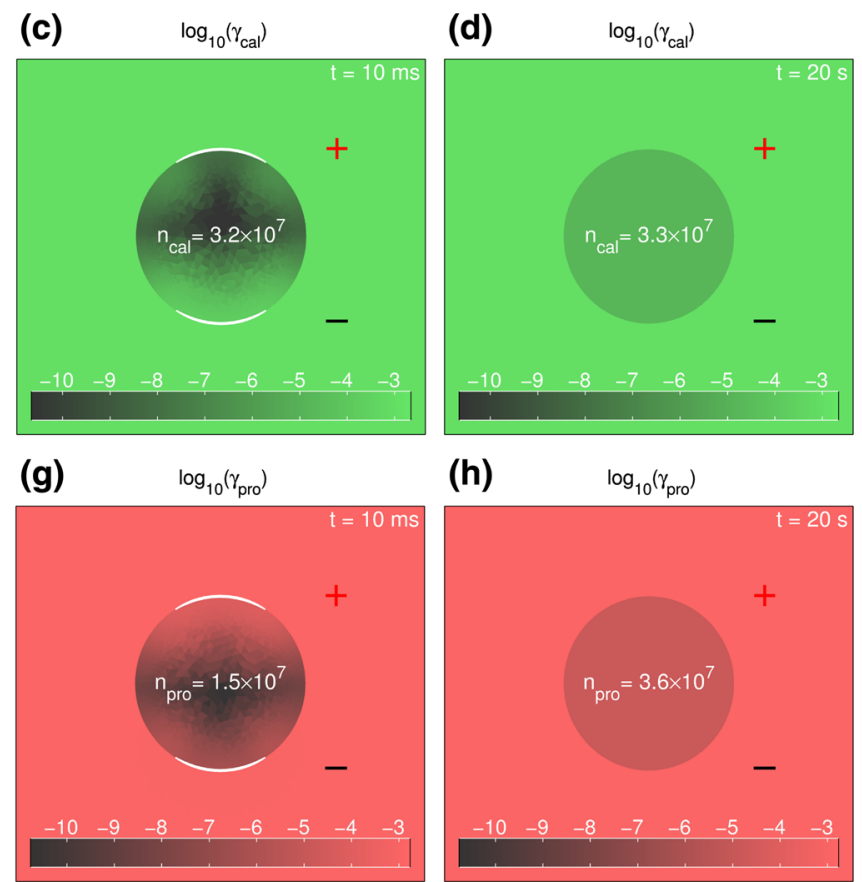

observed during the pulse $(\mathbf{a}, \mathbf{b})$, with only a small amount of diffusional influx occurring after the pulse. The internalization of propidium during the pulse is only about half that of calcein during the pulse, but a larger amount of diffusional influx occurs after the pulse such that roughly equivalent amounts of calcein and propidium are internalized over the $20 \mathrm{~s}$ simulation time (Color figure online)
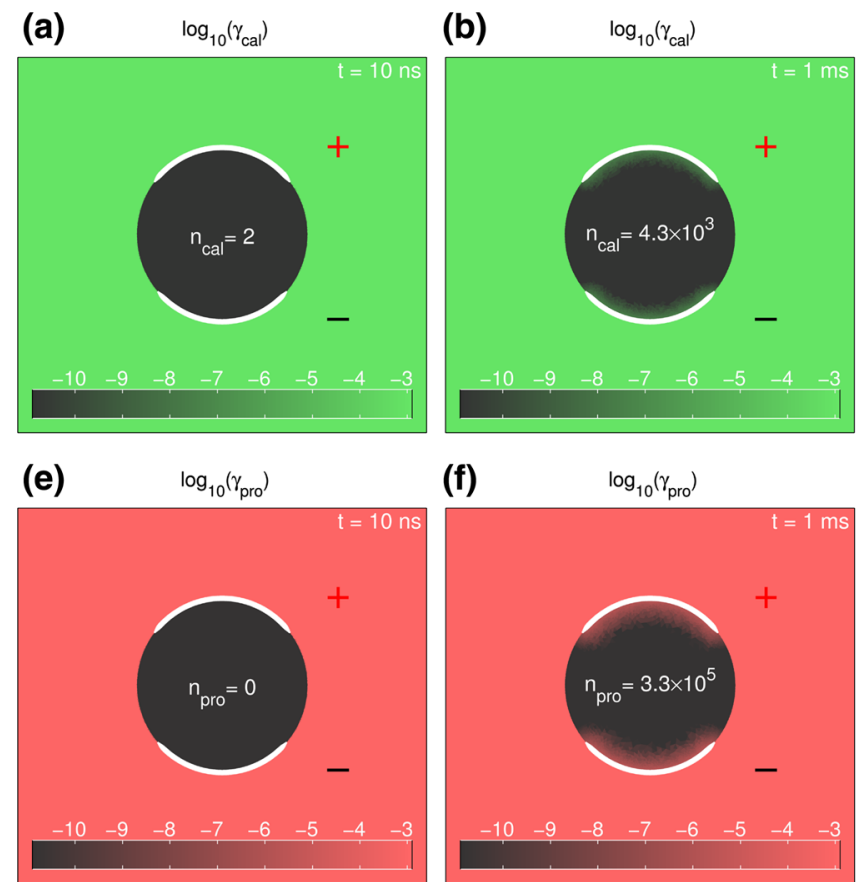

Fig. 5 The logarithm of calcein and propidium concentrations, $\gamma_{\text {cal }}$ and $\gamma_{\text {pro }}$, is shown at $t=10 \mathrm{~ns}(\mathbf{a}, \mathbf{e}), t=1 \mathrm{~ms}(\mathbf{b}, \mathbf{f}), t=105 \mathrm{~ms}(\mathbf{c}, \mathbf{g})$, and $t=20 \mathrm{~s}(\mathbf{d}, \mathbf{h})$ for the $40 \mathrm{kV} / \mathrm{cm}, 10 \mathrm{~ns}$ pulse. The total intracellular amounts of each solute are displayed inside the cell. Red '+' and black '-' indicate the anodic and cathodic sides of the (c)

$\log _{10}\left(\gamma_{\text {cal }}\right)$

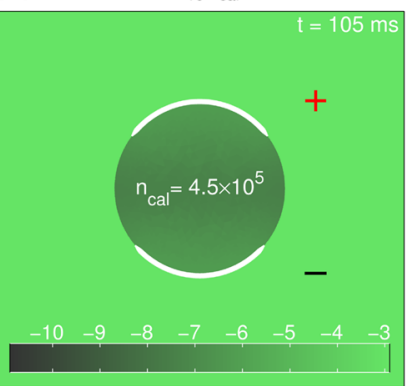

(g)

$\log _{10}\left(\gamma_{\text {pro }}\right)$

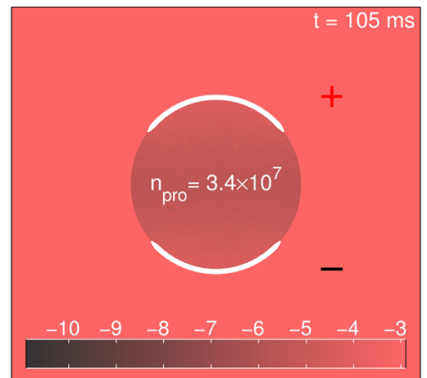

(d) $\quad \log _{10}\left(\gamma_{\text {cal }}\right)$

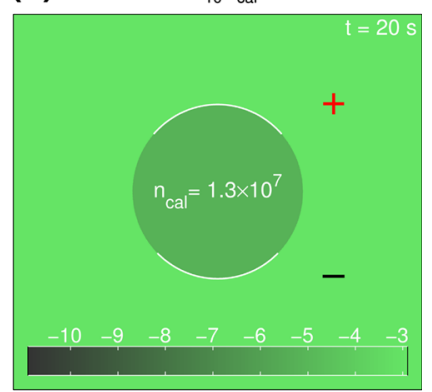

(h)

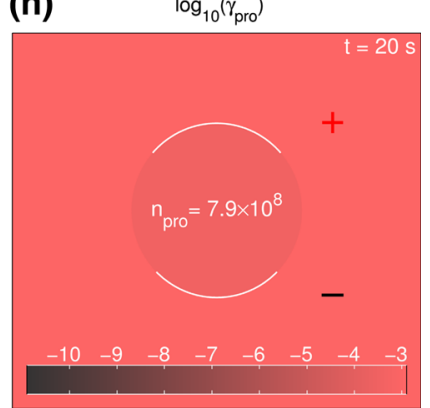

system, respectively. Insignificant amounts of both calcein and propidium are internalized during the pulse $(\mathbf{a}, \mathbf{e})$. However, the large number of pores created allows for much greater amounts of post pulse diffusion compared to the $100 \mu$ s pulse (Color figure online) 
Fig. 6 Electrodiffusive transport rates of propidium and calcein, $d n_{s} / d t$, into the cell for a $1.5 \mathrm{kV} / \mathrm{cm}, 100 \mu$ s pulse on two different timescales $(\mathbf{a}, \mathbf{b})$. Cumulative solute influx, $n_{s}(t)$ $(\mathbf{c}, \mathbf{d})$ shows the total amount of solute inside the cell as a function of time. During the $1.5 \mathrm{kV} / \mathrm{cm}, 100 \mu$ s pulse, $3.2 \times$ $10^{7}$ molecules of calcein and $1.5 \times 10^{7}$ molecules of propidium are transported into the cell. After the $100 \mu$ s pulse, $3.0 \times 10^{5}$ molecules of calcein and $2.1 \times 10^{7}$ molecules of propidium are transported into the cell
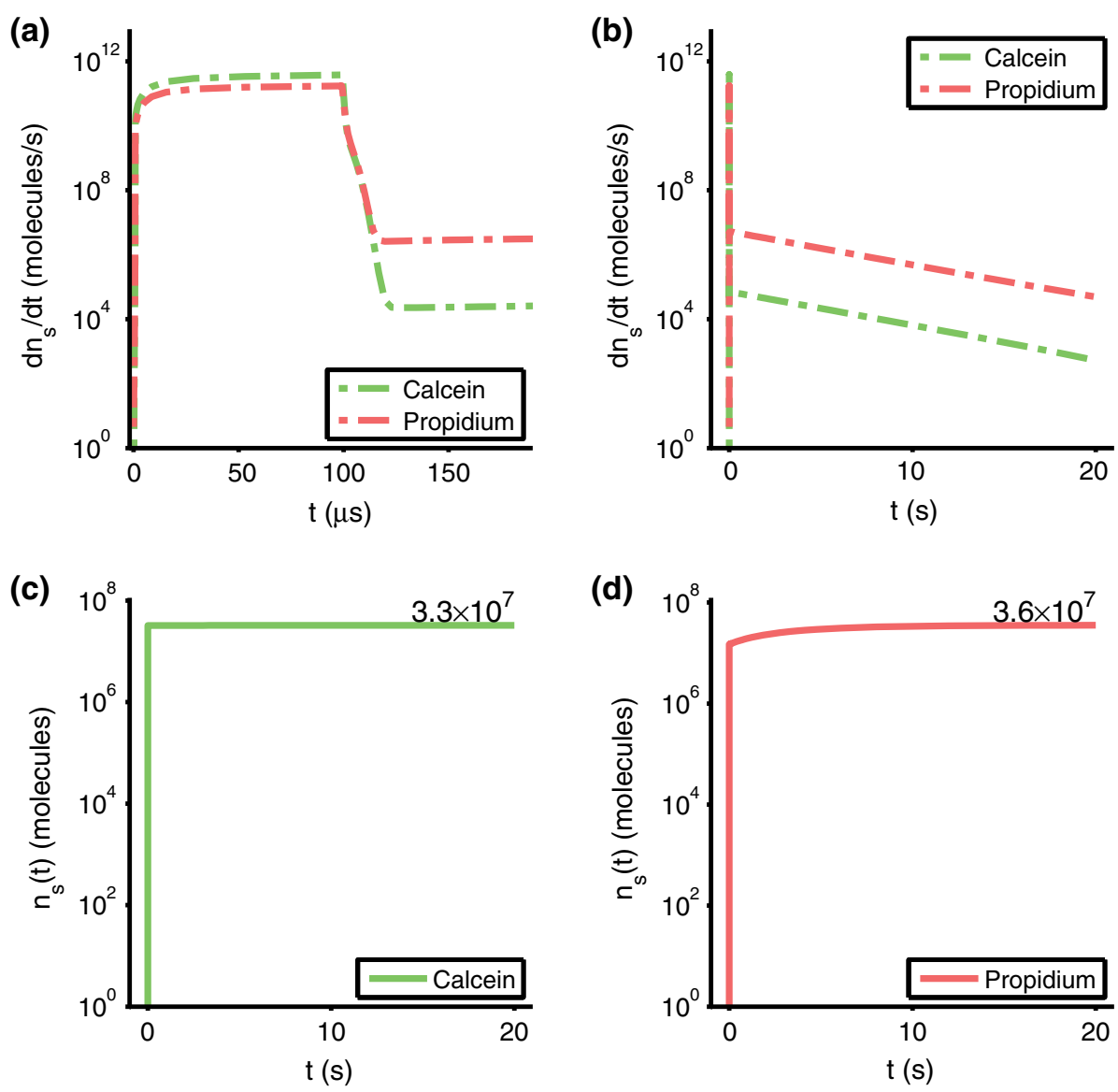

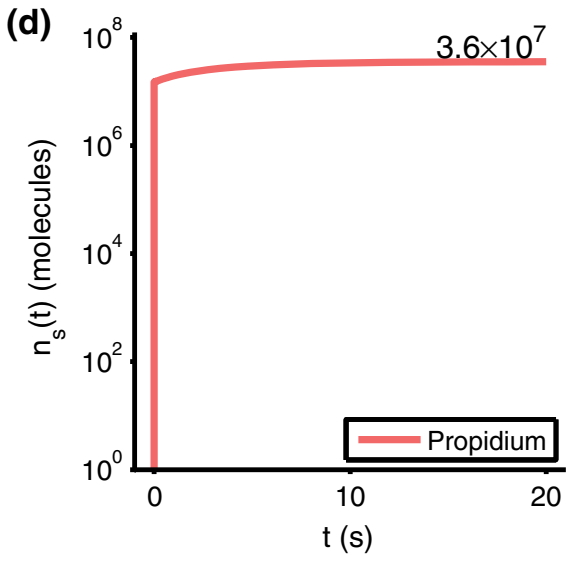

$\gamma_{c a l, i} \leq 10^{-10} \mathrm{M}(0.1 \mathrm{nM})$, i.e., essentially zero (Fig. 4a). At pulse end $(100 \mu \mathrm{s})$, calcein concentration appears as a green fuzzy curved band near the membrane on the cathodic side of the cell (Fig. 4b). This is a diffusively broadened distribution of calcein that has entered mainly by drift from the cathodic side, expected for negatively charged calcein. Close examination reveals a much thinner green band near the membrane around the anodic pole, with two small green areas away from the anodic polethese areas are transition regions that favor large pores (DeBruin and Krassowska 1999a; Krassowska and Filev 2007; Smith 2011). Small amounts of calcein can enter pores and diffuse through the membrane as $\Delta \phi_{\mathrm{m}}$ ramps down before the pulse end. This type of detailed behavior is expected from the model.

Internalized calcein is redistributed by diffusion within the cell, which smooths the intracellular concentration distribution. This is shown by the progression of fuzzy green within the cell at $10 \mathrm{~ms}$ (Fig. 4c). The difference between Fig. $4 \mathrm{~b}$ at $100 \mu$ s (intracellular green near membrane, particularly on the cathodic side) and Fig. 4c (intracellular green spread out by diffusion to clearly mark two anodic locations associated with transition regions) is striking. The visual result of transition regions is a fuzzy upside down black " $\mathrm{T}$ " that occupies the upper half of the cell. By the end of the simulation at $20 \mathrm{~s}$ (Fig. 4d), diffusion has smoothed the calcein distributions, so the extraand intracellular regions are uniformly green. But the total uptake of $n_{\text {cal }}=3.3 \times 10^{7}$ calcein molecules corresponds to a concentration somewhat smaller than the extracellular concentration, which is why the cell interior is darker green than exterior (Fig. 4d).

Overall, calcein enters by electrodiffusion through the dynamic pore populations (Fig. 3a-d). No significant calcein concentration gradients are seen near the cell membrane on either side. This means that the calcein gradient is mostly within the membrane, consistent with even the porated membrane presenting the dominant barrier to solute transport. Also, consistent with a significant porated membrane barrier, calcein uptake results show that only slight additional transport occurs by diffusion after the pulse (Figs. 4c, d, 6).

\section{Propidium Uptake for the $1.5 \mathrm{kV} / \mathrm{cm}, 100 \mu$ s Pulse}

Figure $4 \mathrm{e}-\mathrm{h}$ shows corresponding behavior for propidium (red). As with calcein Fig. 4e shows the initial zero concentration (black). Figure $4 \mathrm{f}$ at $100 \mu$ s shows a curved red 
Fig. 7 Electrodiffusive transport rates of propidium and calcein, $d n_{s} / d t$, into the cell for a $40 \mathrm{kV} / \mathrm{cm}, 10 \mathrm{~ns}$ pulse (a, b). Cumulative solute influx, $n_{s}(t)$ (c, d) shows the total amount of solute inside the cell as a function of time. During the $40 \mathrm{kV} / \mathrm{cm}, 10 \mathrm{~ns}$ pulse, 1.5 molecules of calcein and 0.17 molecules of propidium are transported into the cell. After the $10 \mathrm{~ns}$ pulse, $1.3 \times 10^{7}$ molecules of calcein and $7.9 \times$ $10^{8}$ molecules of propidium are transported into the cell
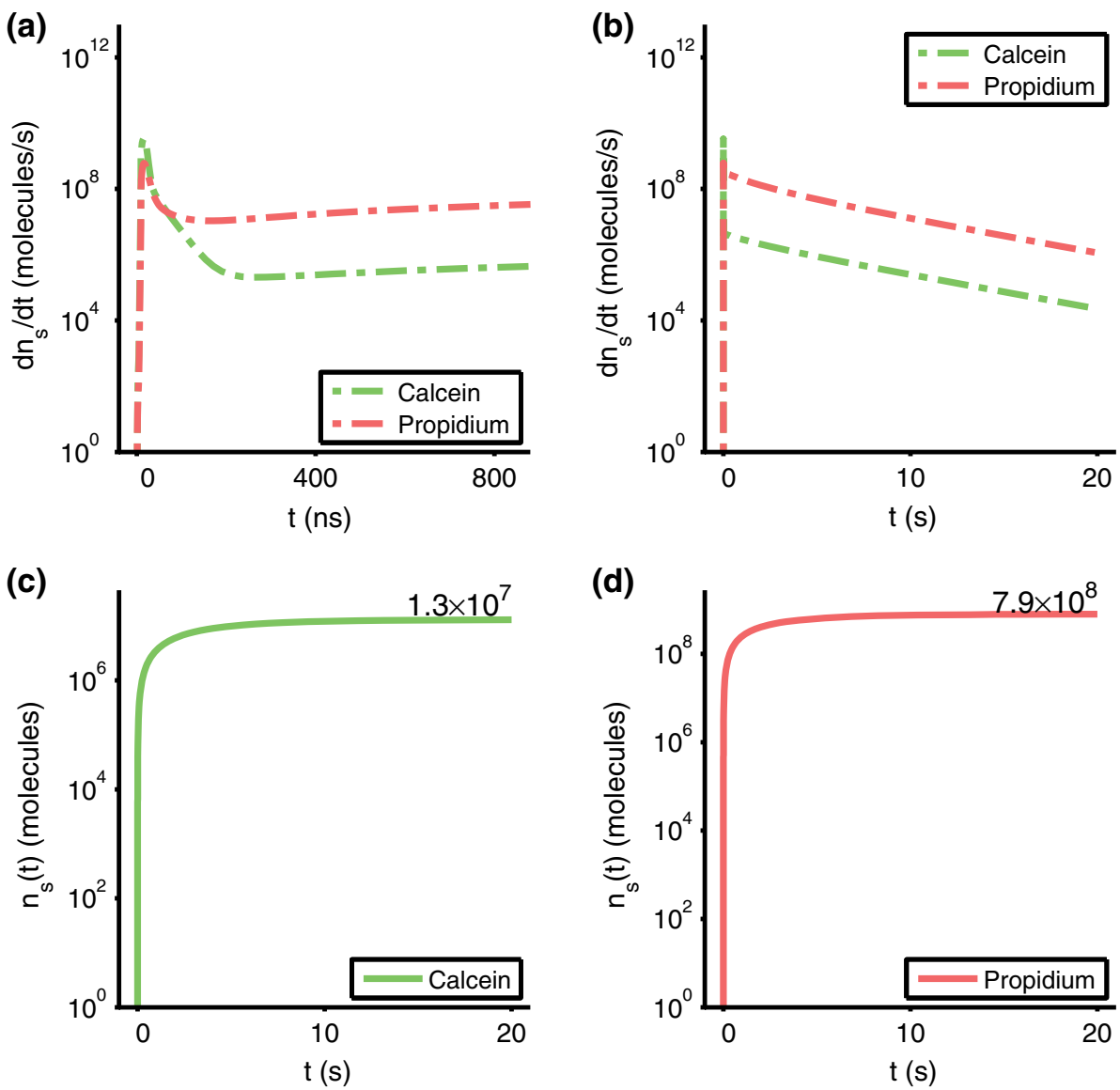

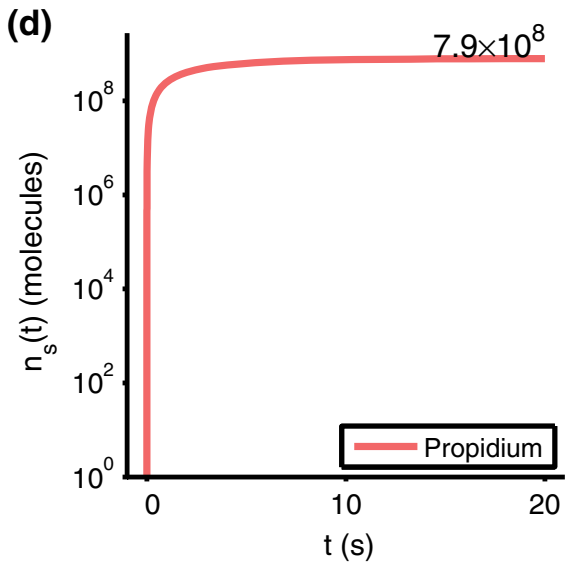

band on the anodic side (top), and only a small hint of entry on the cathodic side (bottom). Here $1.5 \times 10^{7}$ molecules are internalized, about half that for calcein (Fig. 4b). This is consistent with drift-dominated electrodiffusion during the pulse. While nearly the same molecular mass, propidium has a charge number magnitude, $|z|$ of 2 , half that of calcein, consistent with accumulating about half the amount as calcein during the pulse.

Strikingly, comparison of Fig. 4f at $100 \mu$ s, the pulse end, with Fig. $4 \mathrm{~g}$ at $10 \mathrm{~ms}$, show no change during this small portion of the post pulse time. This interval is only $5 \times 10^{-4}$ of the $20 \mathrm{~s}$ post pulse interval. Figure 4 shows that during the $100 \mu \mathrm{s}$ pulse $1.5 \times 10^{7}$ molecules are internalized, but that during the entire post pulse period an additional $2.1 \times 10^{7}$ molecules are taken up. For the model's 4 s pore lifetime, less than $1 \%$ of the pores remain at $20 \mathrm{~s}$, a time at which post pulse transport is essentially complete. The cumulative transport of $3.6 \times 10^{7}$ molecules at $20 \mathrm{~s}$ (Fig. 4h), is a significant increase from $1.5 \times$ $10^{4}$ molecules at $10 \mathrm{~ms}$ (Fig. $4 \mathrm{~g}$ ). Figure $4 \mathrm{~d}$ shows about the same number $\left(3.3 \times 10^{7}\right.$ molecules $)$ for calcein.

The model shows that transport mechanisms for calcein and propidium differ significantly for the $1.5 \mathrm{kV} / \mathrm{cm}$, $100 \mu$ s conventional EP pulse. Almost identical total uptake occurs for calcein (Fig. 4a-d) and propidium (Fig. 4e-h), but is accomplished differently. Calcein is internalized almost entirely by drift during the pulse, with only slightly more $\left(1 \times 10^{6}\right.$ molecules $)$ after $100 \mu$ s. In contrast, propidium is internalized about half by drift during the pulse and half by diffusion after the pulse. See also Fig. 6 .

\section{Calcein Uptake for the $40 \mathrm{kV} / \mathrm{cm}, 10 \mathrm{~ns}$ Pulse}

The nsPEF pulse molecular uptake response is quite different, revealed by both uptake rates (Fig. 7a, b) and cumulative amounts (Fig. 7c, d). A general prediction is that nsPEF creates many more but small pores (Fig. 3e-h) than conventional EP pulses (Fig. 3a-d). Insignificant transport occurs during the pulse due to its very short duration, and post pulse diffusion accounts for essentially all transport (Smith and Weaver 2011).

Figure 5e-h supports this expectation. At the end of pulse (10 ns), only 2 calcein molecules have entered the cell. Essentially all calcein transport occurs post pulse, here at $t=20 \mathrm{~s}$, when $99 \%$ of the pores have vanished. Highly charged calcein should experience diminished entry into a pore because of electrostatic energy cost to insert a charged solute into the narrow interior region of a pore (Parsegian 
1969, 1975; Smith 2011). Nevertheless some calcein does enter by partitioning, and then moves through pores by sterically hindered diffusion (Smith 2011; Smith and Weaver 2011). As the thermalized pore population (Fig. $3 \mathrm{~g}, \mathrm{~h}$ ) decays there is a diffusive calcein influx that also decays with a $4 \mathrm{~s}$ lifetime, leading to cumulative intracellular calcein uptake of $4.3 \times 10^{3}, 4.5 \times 10^{5}$, and $1.3 \times 10^{7}$ molecules at the post pulse times shown in Fig. $5 \mathrm{a}-\mathrm{d}$.

\section{Propidium Uptake for the $40 \mathrm{kV} / \mathrm{cm}, 10 \mathrm{~ns}$ Pulse}

Uptake of propidium is similar, but larger, owing mainly to its twofold smaller charge number and the dominance of post pulse diffusion. Figure 5e shows zero internalized molecules at the pulse end (10 ns). The subsequent entry is similar qualitatively but not quantitatively. The times shown are the same as for calcein (Fig. 5a-d), but the cumulative propidium uptake numbers are larger: $0,3.3 \times$ $10^{5}, 3.4 \times 10^{7}$, and $7.9 \times 10^{8}$ molecules. By $20 \mathrm{~s}$ inspection shows that the intra- and extracellular concentrations of propidium are almost identical (Fig. 5h). The model, however, does not take into account binding of propidium to numerous binding sites within the cell (Sadik et al. 2013). Our present model therefore underestimates propidium uptake, because if binding was included then the intracellular concentrations (free solute) would be smaller, and the gradient across the membrane would be larger. In our present model, the uptake of both calcein and propidium is not that different. The more interesting observation is the relative contribution during a pulse $\left(\Delta \phi_{\mathrm{m}}\right.$ is elevated, supporting drift) and after $\left(\Delta \phi_{\mathrm{m}}=0\right.$, supporting diffusion over a much longer time).

\section{Total Cell Transmembrane Solute Transport Rates}

During a pulse, both $\Delta \phi_{\mathrm{m}}(t)$ and electric fields within the bulk media are elevated. At each membrane node-pair the local $\Delta \phi_{\mathrm{m}}$ creates heterogeneous local fields near the entrance/exits of individual pores, and also within the pore interior. Both are included in the model. These pore-associated fields mostly determine electrodiffusive solute transport, as the fields in bulk away from the membrane are much smaller. That is, electrodiffusion through the individual pores of each population is rate limited, with bulk media fields serving mainly to supply and removing solute from the membrane regions. Even for the highly mobile small ions that govern $\Delta \phi_{\mathrm{m}}$ during supra-EP the porated membrane is the main barrier.

After a pulse the membrane becomes depolarized in about a microsecond. Post pulse solute transport occurs by diffusion that is sterically hindered within pores of a thermalized pore population. Entry into pores is governed by partitioning, which increases as solute charge decreases. Within simple bulk media away from the membrane partitioning is a nonissue, and both diffusion and drift are unhindered.

Calcein and Propidium Influx Rates for the $1.5 \mathrm{kV} / \mathrm{cm}$, 100 us Pulse

Figure 6a shows the calcein influx rate (green dot-dash curve) jumping to $\sim 2 \times 10^{8}$ molecules/s in a few microseconds. But at $100 \mu$ s (pulse end), the rate plummets by more than four orders of magnitude (Fig. 6a), as $\Delta \phi_{\mathrm{m}}$ drops from $\sim 0.5 \mathrm{~V}$ to zero (Fig. $2 \mathrm{a}$ ). The entire cell membrane is then depolarized. With $\Delta \phi_{\mathrm{m}}=0$ solute drift ceases, and post pulse solute transport is by diffusion through the thermalized pore population (Fig. 3d). Similar behavior occurs for propidium (red dot-dash curve). The driftdominated propidium transport during the pulse is slightly smaller owing to its smaller charge number, but is larger post pulse due to more partitioning into pores.

Figure $6 a, b$ both show a rapid rate decrease at the pulse end at $100 \mu \mathrm{s}$. After the pulse there is transient, complicated behavior while the membrane is discharging, and the solute transport is transitioning from drift-dominated to diffusion dominated. This transition interval involves general pore contraction (Fig. 3c, d) as $\Delta \phi_{\mathrm{m}}$ decreases in two phases from 99 to $\sim 101 \mu$ s (Fig. 2a). After this brief transition, both calcein and propidium are transported over the next $20 \mathrm{~s}$ by an exponentially decaying diffusion rate (Fig. 6a, b). The very different rates during and after the pulse are readily seen in Fig. 6b, where the red/green spike (drift) near $t=0$ is followed by two parallel dot-dash lines (diffusion) on this semi-log plot. The details of partitioning and steric hindrance are used in all cases of electrodiffusion through pores of different sizes in each population, and these rates appropriately match those in the nearby bulk media, a basis for the model's solutions being computationally nontrivial.

\section{Calcein and Propidium Influx Rates for the $40 \mathrm{kV} / \mathrm{cm}$, 10 ns Pulse}

The $40 \mathrm{kV} / \mathrm{cm}, 10 \mathrm{~ns}$ pulse has very different solute transport rates (Fig. 7a, b). The calcein and propidium rates rapidly increase from 1 molecule/s (log scale constraint) to a peak values of order $10^{9}$ molecules/s at about 9 ns (peak pulse value; when total pore number, $N$, is reached Fig. $2 \mathrm{j}$ ), and then falling by several orders of magnitude by $400 \mathrm{~ns}$. As shown by the behavior in Fig. 7b-d, these huge rates for very short times are unimportant, given the overwhelming contribution of post pulse diffusion (Fig. 7c, d). 
Calcein and Propidium Cumulative Uptake for the $1.5 \mathrm{kV} /$ cm, 100 us Pulse

Total uptake numbers for calcein $\left(3.3 \times 10^{7}\right.$; Fig. $\left.6 \mathrm{c}\right)$ and propidium $\left(3.6 \times 10^{7}\right.$; Fig. $\left.6 \mathrm{~d}\right)$ are almost the same for this conventional EP pulse, but the contributions during and after the pulse are quite different. During the pulse, $3.2 \times$ $10^{7}$ calcein molecules and $1.5 \times 10^{7}$ propidium ions are internalized, essentially the same within a factor of two. After the pulse the contributions are quite different, only $3.5 \times 10^{5}$ calcein molecules, but $2.1 \times 10^{7}$ propidium ions (Fig. 6c, d).

\section{Calcein and Propidium Cumulative Uptake for the $40 \mathrm{kV} /$ cm, 10 ns Pulse}

Pore creation is complete within only $\sim 9$ ns (Fig. $2 \mathrm{j}$ ). In this very short time drift dominates for both calcein and propidium (Fig. 7c), with diffusion taking over after a transition involving pore contraction and membrane depolarization. Although there are noticeable transport changes between 9 and about 300 ns (Fig. 7b), the contribution to cumulative transport in this interval is negligible, being overwhelmed by the post pulse diffusive contribution (Fig. 6e-h). The importance of post pulse diffusion is consistent with cumulative calcein uptake of $1.3 \times 10^{7}$ molecules, about 70 -fold less than the $7.9 \times$ $10^{8}$ molecules for propidium, which can partition more readily into the interiors of the small pores that comprise the post pulse thermalized pore populations. The predicted dominance of diffusion for total uptake by nsPEF (Smith and Weaver 2011) is consistent with the model's behavior.

The detailed discussion of solute uptake rates and amounts show that cell membrane EP behavior is complex. This complexity is a result of many interacting membrane sites, using a model for dynamic EP at each of the 600 sites. Once established cell EP models with irregular and multiple membranes can be used with confidence, if comparison to experiments are also pursued.

\section{Future Directions}

Modeling should confront three phases of EP phenomena, which involve different time scales and also different types of membrane interactions. The first is pore creation, which can be approached by both continuum models and MD simulations. The second is pore population evolution based on interactions that tend to expand or contract individual pores within a population. The third is post pulse behavior, when the membrane is believed fully depolarized shortly after a pulse. In the absence of an applied field, pore behavior may be affected by other interactions such as intracellular field sources that extend through the porated cell membrane, time-dependent membrane tension from cell swelling, and lipid alteration by interaction with products of stimulated reactions (e.g., reactive oxygen species; ROS) due to EP.

Modeling during the first and second phases involves large fields, and causes changes in $\Delta \phi_{\mathrm{m}}(\mathrm{t})$. Modeling during the third phase is much more challenging, and has received little attention. Fields and transmembrane voltages are small to zero. Candidate interactions are of different types (mechanical, chemical, and thermal), and may involve specificity based on pore constituents and also the transported ions and molecules (solutes). For this reason, and outlined below, we identify new approaches that can extend the present model's capability to address these problems.

The present model is based on well known passive electrical properties of aqueous media and the fixed portions of cell membranes. Typical values from the literature are used. More composition-specific properties could be used, but the present state of quantitative information from cell EP experiments is not good enough to support detailed testing and validation. Nevertheless, as noted above this and other members of a family of cell models can account for several key experimental observations. Below we briefly note extensions of the present model which should bring improved realism and quantification.

\section{Extrapolation of MD Structure-Function Results to Cell Models}

Continuum cell EP models developed over several decades feature homogeneous representations of membrane properties. Even if more than one membrane is included to represent one or more organelles, the individual membranes at most have different homogeneous properties for the unaltered membrane regions, with poration represented by adding highly nonlinear, hysteretic pore models (e.g., Fig. 1a, b). In essence, the traditional Schwan model is extended by adding relatively simple representations of dynamic pores that change in size and conductance as $\Delta \phi_{\mathrm{m}}$ varies with time and position. This is the basis of the present model (Smith 2011; Smith et al. 2013), which includes an unusually complex representation of dynamic pores.

Going forward, MD can provide a further, larger increase in realism using atomistic and molecular constructions, presently of the lipid bilayer regions of a cell membrane. MD examines the response of a small cell membrane patch, initially emphasizing lipids, and within which typically a single, dynamic pore typically appears with both fluctuations and overall expansion and contraction responding to $\Delta \phi_{\mathrm{m}}$. MD studies are increasingly enabled by increasing 
computing power (hardware and software), and the knowledge accumulating from a decade of EP simulations (Tieleman et al. 2003; Böckmann et al. 2008; Levine and Vernier 2010; Delemotte and Tarek 2012; Bennett et al. 2014). MD simulations are relevant to several major and inter-related predictions of the cell model, such as pore creation and lifetimes, pore density, and pore radius. We expect longer simulated times $(\mu \mathrm{s})$, larger simulated areas $\left(\mu \mathrm{m}^{2}\right)$, and more complex membranes (saturated and unsaturated glycerophospholipids, sphingolipids, and cholesterol) for electric field strengths corresponding to porating transmembrane voltages of $\sim 0.5 \mathrm{~V}$ or more. The incorporation of proteins, with molecular detail, will soon be possible. This offers the prospect of including lateral and transverse mechanical constraints imposed by membrane-associated proteins on the restructuring of the lipid bilayer to form a porated complex.

Finally, one specific connection between MD and continuum models, and associated experimental observations is the transport of small solutes through porated membranes. MD simulations can generate conductance and poration results for comparison to results from both continuum cell models and experiments. Quantitative comparisons will improve cell models and will result in a deeper understanding of the existing literature of measured values, and can stimulate and guide new experiments that will support and extend measurement methods and applications.

\section{Post Pulse Landscape-Altering Mechanical} and Chemical Interactions

We hypothesize that the pore energy landscape can change after the pulse, when $\Delta \phi_{\mathrm{m}} \approx 0$. More specifically, we suggest that the effective membrane tension may be significantly altered for cell membranes after field pulses, for durations of seconds to minutes.

An example of membrane tension change is porationinduced osmotic swelling. Large intracellular molecules cannot cross the porated membrane, and therefore create an osmotic gradient, and water diffuses into the cell to restore osmotic equilibrium. Cells respond to this osmotic imbalance in different ways. Some swell, resulting in a volume increase, and the extent and rate of swelling can be correlated to the extent and rate of ion concentration equilibration. The small ion flux that comes at the beginning of this process can be described in detail by MD, enabling a quantitative comparison between ion transport in the models and the swelling in cells.

Similarly, a candidate for poration-induced chemical alteration is reactive species (ROS) generation, in which ROS progressively alters the lipids of the membrane both during and after a pulse. Such chemically-induced changes can also alter the effective membrane tension, changing the landscape, and possibly also the effective pore diffusion coefficient. Relatively little work has been done on either mechanical or chemical interactions that could alter pore behavior after a pulse. Both are attractive general candidates for extending cell EP modeling capability.

\section{Summary}

We present illustrative responses of our present cell EP model, which consists of a closed curved cylindrical membrane that separates two aqueous media. The model is constructed from well known passive electrical properties (conductive and dielectric) of water-based media and also the fixed properties of a cell membrane. The active constituent of the model is a 1D model for membrane EP with dynamic pores. It is a recent version of a family of EP models that feature two rate equations, one for stochastic pore creation, the other for random pore destruction. These two equations introduce or remove pores from a pore energy landscape that changes locally with transmembrane voltage, $\Delta \phi_{\mathrm{m}}(t)$. The various local models are connected by a meshed network that has 1D models for charge transport, forming a system model.

The model is a hypothesis which can be tested by subjecting the model to various stimuli, here two very different electric field pulses. Although these pulses are idealized trapezoids, digitized representations of actual experimental pulses can also be used. The model is complex, but nowhere near the complexity of a real cell membrane. However, the existing capabilities and the prospect for significant extensions together suggest that cell level modeling is an important complement to the many experimental studies of electroporation. A collaborative effort involving coordinated experiments and models could significantly accelerate basic studies, and also begin to set the stage for personalized models for tumor treatments, in which various imaging modalities aid model construction and biochemical information from circulating tumor cells may allow development of cell EP models that target cancer cells and tumors to improve treatments. Both EPbased drug delivery (ECT using bleomycin or cisplatin, or calcium EP) or drug-free, nonthermal ablation by IRE or nsPEF should benefit from this approach that begins to set the stage for a more focused biomedical engineering based on electroporation.

\section{Appendix: Two Closely Related, Simple Passive Cell Models}

While referring to all other underlying equations through citations that provide mathematical details and 
explanations, here we present two versions of a basic passive cell model equation, often referred to as the Schwan equation in the spherical case (Pauly and Schwan 1959; Kotnik and Miklavčič 2000; Foster 2000; Kotnik and Miklavčič 2006). The above papers contain considerable detail, with different approximations, and these include the transient responses of the two models. This simplest version is for the case that the passive cell membrane becomes fully charged. Then, fields and transmembrane voltages are no longer changing, and this direct current $(\mathrm{dc})$ version is often presented. The two simplest versions are

$$
U_{\mathrm{m}, \mathrm{sph}}=\frac{3}{2} E_{\mathrm{e}} r_{\mathrm{cell}} \cos \theta
$$

Spherical membrane Schwan equation

$$
\begin{aligned}
U_{\mathrm{m}, \mathrm{cyl}}= & 2 E_{\mathrm{e}} r_{\text {cell }} \cos \theta \\
& \text { Cylindrical membrane Schwan equation }
\end{aligned}
$$

Here the notation is $E_{\mathrm{e}}$ for the magnitude of the uniform field within the extracellular aqueous medium that fills the simulation box (entire system model) with no cell present. $E_{\mathrm{e}}$ can differ significantly from the nominal field, $E_{\mathrm{n}}$, a potential source of error if not accounted for (Pliquett et al. 1996). This simple comparison shows that the $2 \mathrm{D}$ cylindrical version has the same form, with the induced transmembrane voltage differing only by a factor $4 / 3$. The two fields can be essentially the same for some pulsing media and electrode composition, but for others the electrolyte field can be smaller, e.g., $90 \%$ of the nominal strength (Canatella et al. 2001). This difference can be significant in the "standard model" given the $\Delta \phi_{\mathrm{m}}^{2}$ dependence in the creation rate equation. Our model assumes $E_{\mathrm{e}}(\mathrm{t})$, the actual field within the electroporating medium.

This simplest case has zero resting potential (strictly resting transmembrane voltage, $\Delta \phi_{\mathrm{m}, \text { rest }}$ ). These equations are consistent with field amplification (Fig. SI-2). We use the version that yields the transmembrane voltage at each membrane site, with the resting potential included through assignment of a nonequilibrium source of the resting potential (Läuger 1991; DeBruin and Krassowska 1999a).

Acknowledgments Supported by NIH grant GM063857 to JCW, and NSF and HST fellowships to KCS. PTV supported in part by the Air Force Office of Scientific Research and the University of Southern California Center for High Performance Computing and Communications. We thank A. Pakhomov and H. Lin for multiple stimulating discussions, D. Miklavcic and L. Mir for organizing the EBTT workshop where some of this was presented, and K. G. Weaver for continued computer support. We apologize to our many colleagues for not being able to cite all of their important work that is relevant, but space limitations prevent inclusion of a comprehensive review section.

\section{References}

Abidor IG et al (1979) Electric breakdown of bilayer membranes: I. The main experimental facts and their qualitative discussion. Bioelectrochem Bioenerg 6:37-52

Barnett A, Weaver JC (1991) Electroporation: a unified, quantitative theory of reversible electrical breakdown and rupture. Bioelectrochem Bioenerg 25:163-182

Beebe SJ et al (2002) Nanosecond pulsed electric field (nsPEF) effects on cells and tissues: apoptosis induction and tumor growth inhibition. IEEE Trans Plasma Sci 30:286-292

Beebe SJ et al (2003) Nanosecond, high intensity pulsed electric fields induce apoptosis in human cells. FASEB J 17:1493-1495

Benkler S et al (2008) Mastering conformal meshing for complex CAD-based C-FDTD simulations. IEEE Antennas Propag Mag 50:45-56

Bennett WFD et al (2014) Atomistic simulations of pore formation and closure in lipid bilayers. Biophys J 106:210-219

Benz R et al (1979) Reversible electrical breakdown of lipid bilayer membranes: a charge-pulse relaxation study. J Membr Biol 48:181-204

Böckmann RA et al (2008) Kinetics, statistics, and energetics of lipid membrane electroporation studied by molecular dynamics simulations. Biophys J 95:1837-1850

Breton M, Mir LM (2012) Microsecond and nanosecond electric pulses in cancer treatments. Bioelectromagnetics 33:106-123

Canatella PJ et al (2001) Quantitative study of electroporationmediated molecular uptake and cell viability. Biophys $\mathrm{J}$ 80:755-764

Chernomordik LV et al (1987) The electrical breakdown of cell and lipid membranes: the similarity of phenomenologies. Biochim Biophys Acta 902:360-373

Christ A et al (2010) The "Virtual Family" Project: development of anatomical whole-body models of two adults and two children. Comput Med Biol 55:23-38

Craviso GL et al (2012) Modulation of intracellular $\mathrm{Ca}^{2+}$ levels in chromaffin cells by nanoelectropulses. Bioelectrochemistry 87:244-252

Davalos RV et al (2004) Electrical impedance tomography for imaging tissue electroporation. IEEE Trans BME 51:761-767

Davalos RV et al (2005) Tissue ablation and irreversible electroporation. Ann Biomed Eng 33:223-231

DeBruin KA, Krassowska W (1999a) Modeling electroporation in a single cell: I. Effects of field strength and rest potential. Biophys J 77:1213-1224

DeBruin KA, Krassowska W (1999b) Modeling electroporation in a single cell: II. Effects of ionic concentration. Biophys J $77: 1225-1233$

Delemotte L, Tarek M (2012) Molecular dynamics simulations of lipid membrane electroporation. J Membr Biol 245:531-543

Dev SB et al (2000) Medical applications of electroporation. IEEE Trans Plasma Sci 28:206-223

Elia $S$ et al (2010) Influence of uncertain electrical properties on the conditions for the onset of electroporation in an eukaryotic cell. IEEE Trans Nanobiosci 9:204-212

Esser AT et al (2010) Mechanisms for the intracellular manipulation of organelles by conventional electroporation. Biophys J 98:2506-2514

Esser AT et al (2007) Towards solid tumor treatment by irreversible electroporation: intrinsic redistribution of fields and currents in tissue. Technol Cancer Res Treat 6:261-273

Esser AT et al (2009) Towards solid tumor treatment by nanosecond pulsed electric fields. Technol Cancer Res Treat 8:289-306 
Fernández ML et al (2012) Size-controlled nanopores in lipid membranes with stabilizing electric fields. Biochem Biophys Res Commun 423:325-330

Flickinger B et al (2010) Transmembrane potential measurements on plant cells using the voltage-sensitive dye ANNINE-6. Protoplasma 247:3-12

Foster KR (2000) Thermal and nonthermal mechanisms of interaction of radio-frequency energy with biological systems. IEEE Trans Plasma Sci 28:15-23

Frandsen SK et al (2012) Direct therapeutic applications of calcium electroporation to effectively induce tumor necrosis. Cancer Res $72: 1336-1341$

Frey W et al (2006) Plasma membrane voltage changes during nanosecond pulsed electric field exposures. Biophys J 90:3608-3615

Galluzzi L et al (2012) Molecular definitions of cell death subroutines: recommendations of the Nomenclature Committee on Cell Death 2012. Cell Death Differ 19:107-120

Garcia PA et al (2011) Non-thermal irreversible electroporation (NTIRE) and adjuvant fractionated radiotherapeutic multimodal therapy for intercranial malignant glioma in a canine patient. Technol Cancer Res Treat 10:73-83

Garon EB et al (2007) In vitro and in vivo and a case report of intense nanosecond pulsed electric fields as a local therapy for human malignancies. Int J Cancer 121:675-682

Glaser RW et al (1988) Reversible electrical breakdown of lipid bilayers: formation and evolution of pores. Biochim Biophys Acta 940:275-287

Golberg A, Yarmush ML (2013) Nonthermal irreversible electroporation: fundamentals, applications, and challenges. IEEE Trans Biomed Eng 60:707-714

Gothelf A et al (2003) Electrochemotherapy: results of cancer treatment using enhanced delivery of bleomycin by electroporation. Cancer Treat Rev 29:371-387

Gowrishankar TR et al. (2011). Intracellular electroporation site distributions: Modeling examples for nsPEF and IRE pulse waveforms. In: Proceedings of 33rd Annual Internatinal Conference of IEEE EMBS Boston, MA, Aug 30-Sep 3, 2011. pp 732-735

Gowrishankar TR et al (2006) Microdosimetry for conventional and supra-electroporation in cells with organelles. Biochem Biophys Res Commun 341:1266-1276

Gowrishankar TR, Weaver JC (2003) An approach to electrical modeling of single and multiple cells. Proc Natl Acad Sci 100:3203-3208

Gowrishankar TR, Weaver JC (2006) Electrical behavior and pore accumulation in a multicellular model for conventional and supra-electroporation. Biochem Biophys Res Commun 349:643-653

Gurtovenko AA, Vattulainen I (2007) Ion leakage through transient water pores in protein-free lipid membranes driven by transmembrane ionic charge imbalance. Biophys J 92:1878-1890

Jensen MØ et al (2013) Atomic-level simulation of current-voltage relationships in single-file ion channels. J Gen Physiol 141:619-632

Joshi RP, Schoenbach KH (2010) Bioelectric effects of intense ultrashort pulses. Crit Rev Biomed Eng 38:255-304

Kakorin S, Neumann E (2002) Ionic conductivity of electroporated lipid bilayer membranes. Bioelectrochemistry 56:163-166

Kaner A et al (2013) Model of pore formation in a single cell in a flow-through channel with micro-electrodes. Biomed Microdev 16(2):181-189

Kennedy SM et al (2014) Cationic peptide exposure enhances pulsedelectric-field-mediated membrane disruption. PLOS One 10(1371):0092528
Kinosita K et al (1988) Electroporation of cell membrane visualized under a pulsed-laser fluorescence microscope. Biophys J 53:1015-1019

Kotnik T, Miklavčič D (2000) Second-order model of membrane electric field induced by alternating external electric fields. IEEE Trans Biomed Eng 47:1074-1081

Kotnik T, Miklavčič D (2006) Theoretical evaluation of voltage inducement on internal membranes of biological cells exposed to electric fields. Biophys J 90:480-491

Krassowska W, Filev PD (2007) Modeling electroporation in a single cell. Biophys J 92:404-417

Läuger P (1991) Electrogenic ion pumps. Sinauer Associates, Sunderland

Levine ZA, Vernier PT (2010) Life cycle of an electropore: fielddependent and field-independent steps in pore creation and annihilation. J Membr Biol 236:27-36

Li J, Lin H (2011) Numerical simulation of molecular uptake via electroporation. Bioelectrochemistry 82:10-21

Litster JD (1975) Stability of lipid bilayers and red blood cell membranes. Phys Lett 53A:193-194

Melikov KC et al (2001) Voltage-induced nonconductive pre-pores and metastable pores in unmodified planar bilayer. Biophys $\mathbf{J}$ 80:1829-1836

Merla C et al (2012) Novel passive element circuits for microdosimetry of nanosecond pulsed electric fields. IEEE Trans Biomed Eng 59:2301-2311

Merla C et al (2011) Microdosimetry for nanosecond pulsed electric field applications: a parametric study for a single cell. IEEE Trans Biomed Eng 58(5):1294-1302

Miklavčič D et al (2005) The effect of high frequency electric pulses on muscle contractions and antifumor efficiency in vivo for a potential use in clinical electrochemotherapy. Bioelectrochemistry $65: 121-128$

Miller L et al (2005) Cancer cells ablation with irreversible electroporation. Technol Cancer Res Treat 4:699-705

Mir LM et al (1988) Introduction of definite amounts of nonpermeant molecules into living cells after electropermeabilization: direct access to the cytosol. Exp Cell Res 175:15-25

Müller KJ et al (2001) Reversible electropermeabilization of mammalian cells by high-intensity, ultra-short pulses of submicrosecond duration. J Membr Biol 184:161-170

Nagaoka T et al (2008) Proportion-corrected scaled voxel models for Japanese children and their application to the numerical dosimetry of specific absorption rate for frequencies from 30 $\mathrm{MHz}$ to $3 \mathrm{GHz}$. Phys Med Biol 52:6695-6711

Napotnik TB et al (2012) Nanosecond electric pulses cause mitochondrial membrane permeabilization in Jurkat cells. Bioelectromagnetics 33:257-264

Neu JC, Krassowska W (1999) Asymptotic model of electroporation. Phys Rev E 59:3471-3482

Nuccitelli R et al (2006) Nanosecond pulsed electric fields cause melanomas to self-destruct. Biochem Biophys Res Commun 343:351-360

Nuccitelli R et al (2014) First-in-human trial of nanoelectroablation therapy for basal cell carcinoma: proof of method. Exp Dermatol 23:1933-1939

Pakhomov AG et al (2007a) Long-lasting membrane permeabilization in mammalian cells by nanosecond pulsed electric field (nsPEF). Bioelectromagnetics 28:655-663

Pakhomov AG et al (2007b) Membrane permeabilization and cell damage by ultrashort electric field shocks. Arch Biochem Biophys 465:109-118

Parsegian VA (1969) Energy of an ion crossing a low dielectric membrane: solutions to four relevant electrostatic problems. Nature 221:844-846 
Parsegian VA (1975) Ion-membrane interactions as structural forces. Ann N Y Acad Sci 264:161-174

Pastushenko VF et al (1979) Electric breakdown of bilayer membranes: VI. A stochastic theory taking into account the processes of defect formation and death: membrane lifetime distribution function. Bioelectrochem Bioenerg 6:89-95

Pauly H, Schwan HP (1959) Über die Impedanz einer Suspension von kugelförmigen Teilchen mit einer Schale. Z Naturforsch 14B:125-131

Pliquett $U$ et al (1996) Determination of the electric field and anomalous heating caused by exponential pulses with aluminum electrodes in electroporation experiments. Bioelectrochem Bioenerg 39:39-53

Poddevin B et al (1991) Very high cytotoxicity of bleomycin introduced into the cytosol of cells in culture. Biochem Pharmacol 42:567-575

Puc M et al (2003) Quantitative model of small molecules uptake after in vitro cell electropermeabilization. Bioelectrochemistry 60:1-10

Rems L et al (2013) Cell electrofusion using nanosecond electric pulses. Sci Rep 3:3382-1-3382-10

Retelj L et al (2013) Electroporation of intracellular liposomes using nanosecond electric pulses theoretical study. IEEE Trans Biomed Eng 60:2624-2635

Romeo S et al (2013) Water influx and cell swelling after nanosecond electropermeabilization. Biochim Biophys Acta 1828(8):1715-1722

Rubinsky B (ed) (2010) Irreversible electroporation. Springer, Berlin

Rubinsky J et al (2008) Optimal parameters for the destruction of prostate cancer using irreversible electroporation. J Urol 180:2668-2674

Sadik MM et al (2013) Quantification of propidium iodide delivery using millisecond electric pulses: experiments. Biochim Biophys Acta 1828:1322-1328

Schoenbach KH et al (2001) Intracellular effect of ultrashort pulses. Bioelectromagnetics 22:440-448

Shaw DE et al (2010) Atomic-level characterization of the structural dynamics of proteins. Science 330:341-346

Silve A et al (2012) Demonstration of cell membrane permeabilization to medium-sized molecules caused by a single $10 \mathrm{~ns}$ electric pulse. Bioelectrochemistry 87:260-264

Slepchenko BM et al (2003) Quantitative cell biology with the virtual cell. Trends Cell Biol 13:570-576

Smith KC (2011) A unified model of electroporation and molecular transport. Ph.D. Thesis, Massachusetts Institute of Technology. http://dspace.mit.edu/bitstream/handle/1721.1/63085/725958797. pdf

Smith KC et al (2004) Model of creation and evolution of stable electropores for DNA delivery. Biophys J 86:2813-2826

Smith KC et al (2013) Emergence of a large pore subpopulation during electroporating pulses. Bioelectrochemistry. doi:10.1016/ j.bioelechem.2013.10.009

Smith KC, Weaver JC (2008) Active mechanisms are needed to describe cell responses to submicrosecond, megavolt-per-meter pulses: cell models for ultrashort pulses. Biophys J 95: $1547-1563$

Smith KC, Weaver JC (2011) Transmembrane molecular transport during versus after extremely large, nanosecond electric pulses. Biochim Biophys Res Commun 412:8-12

Smith KC, Weaver JC (2012) Electrodiffusion of molecules in aqueous media: a robust, discretized description for electroporation and other transport phenomena. IEEE Trans Biomed Eng 59:1514-1522

Stewart DA et al (2004) Transport lattice approach to describing cell electroporation: use of a local asymptotic model. IEEE Trans Plasma Sci 32:1696-1708

Sugar IP (1981) The effects of external fields on the structure of lipid bilayers. J Physiol Paris 77:1035-1042

Suzuki DOH et al (2011) Theoretical and experimental analysis of electroporated membrane conductance in cell suspension. IEEE Trans Biomed Eng 58:3310-3318

Talele S et al (2010) Modelling single cell electroporation with bipolar pulse parameters and dynamic pore radii. J Electrost 68:261-274

Tarek M (2005) Membrane electroporation: a molecular dynamics simulation. Biophys J 88:4045-4053

Taupin C et al (1975) Osmotic pressure induced pores in phospholipid vesicles. Biochemistry 14:4771-4775

Tieleman DP (2004) The molecular basis of electroporation. BMC Biochem 5:10

Tieleman DP et al (2003) Simulation of pore formation in lipid bilayers by mechanical stress and electric fields. J Am Chem Soc 125:6382-6383

Vasilkoski Z et al (2006) Membrane electroporation: the absolute rate equation and nanosecond timescale pore creation. Phys Rev E 74:021904

Vernier PT et al (2006a) Nanoelectropulse-driven membrane perturbation and small molecule permeabilization. BMC Cell Biol 7:37-1-37-16

Vernier PT, Ziegler MJ (2007) Nanosecond field alignment of head group and water dipoles in electroporating phospholipid bilayers. J Phys Chem B 111:12993-12996

Vernier PT et al (2006b) Nanopore formation and phosphatidylserine externalization in a phospholipid bilayer at high transmembrane potential. J Am Chem Soc 128:6288-6289

Weaver JC, Chizmadzhev YA (1996) Theory of electroporation: a review. Bioelectrochem Bioenerg 41:135-160

Weaver JC, Mintzer RA (1981) Decreased bilayer stability due to transmembrane potentials. Phys Lett 86A:57-59

Weaver JC et al (2012) A brief overview of electroporation pulse strength-duration space: a region where additional intracellular effects are expected. Bioelectrochemistry 87:236-243

Yao C et al (2005) Analysis of transmembrane potentials induced by pulsed electric field with different durations based on fiveshelled dielectric model of cell. In: Conference of Proceedings of IEEE Engineering in Medicine and Biology Society, pp 4243-4246 